\title{
Syntax and Language Disorders
}

\author{
Martina Penke, U. Gent
}

to appear in: Tibor Kiss, Artemis Alexiadou (eds.): Syntax ( $2^{\text {nd }}$ edition). An International Handbook. Berlin: Walter de Gruyter.

\section{Introduction}

Syntactic deficits are common in language disorders and have always been at the focus of research on language disorders. The investigation whether or not syntactic deficits occur in a given acquired or developmental language disorder, which syntactic structures or processes are eventually affected and how to capture such deficits in an explanatory theoretical account has dominated the linguistic research on language disorders since its very first beginnings to the present.

Interest on syntactic deficits in language disorders first focussed on Broca's aphasia - an acquired language disorder caused by strokes affecting left frontal brain regions. The core symptom of Broca's aphasia is a so-called agrammatic spontaneous speech production which is characterised by omissions of free functional elements and a severe reduction of the length and syntactic complexity of utterances. This leads to a preponderance of very short utterances that seem to consist of a simple, linearly organised string of open-class words. As the term 'agrammatic' already indicates, the spontaneous speech of language impaired speakers suffering from this disorder gives the impression of lacking syntactic structure. Hence, agrammatic Broca's aphasia has been considered the ideal candidate for investigating syntactic deficits and has been at the focus of linguistic investigations concerned with syntactic disorders ever since the advent of modern generative syntactic theory (Chomsky 1957).

According to Chomsky's generative approach, the human language faculty is due to a specialized, domain-specific language organ situated in the brain that is part of our biological endowment and therefore genetically specified (e.g. Chomsky 1980, 2002). If this conception holds true, the language organ in the human brain should be affected by brain lesions involving this organ. If the impaired brain tissue subserves this language organ, the impairment of this organ due to a brain lesion should lead to impairments of the language faculty. Agrammatic Broca's aphasia seemed to exemplify this theoretically predicted case of an impaired language faculty due to brain lesions. Consequently, from the late 1960's to the late 1980's the linguistic investigation of language disorders associated with syntactic deficits was predominantly concerned with the language deficits observed in Broca's aphasia, the focus of this research being on the issue whether or not Agrammatism is due to a deficit affecting syntactic competence and how to capture this purported syntactic-competence deficit in a syntaxtheoretical framework.

Then the discovery of the British KE family, a huge three-generational family in which about half of the members suffer from an inherited speech and language disorder (Gopnik and Crago 1991), drew the attention to developmental language disorders, specifically to the language disorder called Specific Language Impairment $(S L I) .{ }^{1}$ If the language capacity is innately specified, as suggested by Chomsky's generative approach, this capacity should in principle be susceptible to genetic disorders which affect the genetically encoded blueprints controlling the development and functioning of the brain areas that subserve this language organ. Indeed, in 2001 a point-mutation of the human gene FOXP2 (located on the long arm of chromosome 7) 
was found to be related to the inherited speech and language disorder affecting the members of the KE family (Lai et al. 2001). The findings suggest that the mutation of this gene leads to an atypical development of brain areas typically associated with speech and language (Liégeois et al. 2003). This is the first case attested where a genetic defect can be directly correlated to neuro-anatomical abnormalities which in turn are held responsible for certain speech and language functions. FOXP2 was therefore enthusiastically celebrated as the first 'language gene' to be discovered. Meanwhile, further genetic investigations have revealed that the point mutation discovered by the KE family is not regularly observed in other individuals with Specific Language impairment (e.g. SLI Consortium 2002; Meaburn et al. 2002). Nevertheless, Specific Language Impairment has a genetic basis (cf. SLI Consortium 2002; overview in Stromswold 2001) - although it is not yet clear precisely which and how many mutations are involved - and children with Specific Language Impairment display severe problems in acquiring inflectional morphology, verb movement and complex syntactic constructions such as relative clauses or passives. Thus, the deficits associated with Specific Language Impairment have attracted much research on syntactic deficits over the last 20 years.

Besides these two core syndromes, research on syntactic deficits has more recently taken other language disorders such as Down syndrome, Parkinson's syndrome, or Autism into focus. Today, there is hardly an acquired or developmental deficit syndrome associated with language impairments for which a deficit affecting the syntactic domain is not discussed. Such syndromes include developmental language deficits, such as Specific Language Impairment, Williams syndrome, Down syndrome, Autism, or childhood aphasia, and acquired language deficits, such as aphasic language disorders (Broca's aphasia, Wernicke's aphasia) and degenerative brain diseases (Parkinson's disease, Alzheimer's disease, Huntington's disease).

The wealth of research that has been performed in this area up to today precludes any indepth discussion of the investigations conducted and the discussions led - present and past for every single language disorder. I will, therefore, try to single out some major issues that have shaped the field during the last 40 years. Controversies with respect to syntactic deficits have centred on the question whether or not the symptoms related to a given language disorder are due to a deficit affecting syntactic competence and if so, how to capture this deficit in a theoretical framework. Many of the core research issues first arose with respect to Broca's aphasia, the first language disorder to attract linguistically based research, and therefore, research on Broca's aphasia will be at the focus of this article. Nevertheless, the article tries to achieve a broader coverage of the different language disorders by referring to related research conducted on other language disorders when possible and by taking up issues and findings that are specific for single language disorders. In addition, the appendix gives short descriptions of the most relevant language disorders that have been associated with syntactic deficits, sketches the relevance of these disorders for the field and gives references to publications where the reader can find more detailed information on the respective disorder.

\subsection{Why investigating syntactic deficits?}

In investigating language disorders the aim is twofold. A first goal is to describe and explain which aspects of the language faculty are impaired or retained in a given language disorder, i.e. we want to learn about the impaired language system. Such knowledge could then ideally provide the basis for a potential, effective therapeutical intervention. Most research in language disorders has naturally concentrated on this goal. However, although the task is set out precisely enough in theory, accomplishing this task has turned out to be quite intricate in practice, and there is not a single language disorder for which a general consensus regarding 
the nature of the observed language deficits has been obtained among researchers to date (see section 2.).

A second goal of the investigation of syntactic deficits is to learn about the structure of the human language capacity by investigating cases where this capacity seems to break down. Here, the idea is that a thorough study of the syntactic deficits associated with language disorders might provide insights into the structure and organization of the normal language system. Since Chomsky (1957) introduced the generative approach into the field of linguistics, the subject matter of investigation for a generative linguist is the abstract linguistic knowledge of an individual, i.e. his/her language competence or I-language. This knowledge is accessed when we produce respectively comprehend utterances or when we judge their grammaticality. The application of this abstract system of knowledge in language production, comprehension, or judgement results in performance data. The abstract system of grammatical knowledge is not directly accessible to investigation, it remains a black box. Insights in our language competence can only be gained by the analysis of performance data reflecting the application of this knowledge, i.e. when we produce or comprehend speech utterances or judge their grammaticality. Note, however, that grammatical competence is only one factor determining performance, with other factors, such as e.g. processing capacities, social, pragmatic, or discourse factors affecting it, too. As linguistic competence is a 'black box', which does not allow for direct observation, the crucial task for generative linguists is to find evidence for the content of this black box within performance data. The deficits observed in individuals with language disorders constitute a type of performance data that can be used to draw inferences about our language competence. The value of erroneous forms was probably first noticed in speech-error research. A linguistic investigation of speech errors quickly revealed that such errors were not random, but were constrained by the architecture of the language system and only involved elements of the same category and level of representation (Fromkin 1971; Shattuck-Hufnagel 1979). A similar logic has been applied to erroneous forms produced in language impairments, the idea being that we can learn about the human language faculty if we investigate what factors constrain the errors that occur in language impaired individuals (cf. Fromkin 1997 for overview).

The assumption that the investigation of syntactic deficits can provide insights into the structure of the human language faculty rests on four axioms on the nature of the human language capacity and its breakdown following brain lesions. According to the assumptions of autonomy and modularity (Fodor 1983) the human language faculty is autonomous from other cognitive domains or modules (i.e. it is domain-specific) and consists of task-specific and independent submodules carrying out different types of computations on different types of representations. According to the fractionation assumption (Caramazza 1984) brain damage can result in the selective impairment of specific submodules of the language capacity. The transparency assumption (Caramazza 1984) states that the components unaffected by the brain lesion will continue to function as normal such that the output of the language system will directly reflect the impairment of the affected component. The function of the impaired module in the unimpaired language system can, then, be directly inferred from the symptoms observed in the language impaired individual. ${ }^{2}$

In pursuing the goal to draw inferences about the normal language faculty by investigating languages disorders, the investigation of selective deficits, i.e. of deficits that selectively affect a function $\mathrm{X}$ but spare a different function $\mathrm{Y}$, has drawn particular attention. The observation that a linguistic entity or function can be selectively affected in a specific language disorder is seen as evidence that this entity or function is represented in the mind/brain in a way that is distinct from those functions or entities that are unaffected by this language disorder. ${ }^{3}$ Thus, it has been 
suggested, for example, that agrammatic Broca's aphasia is due to a syntactic deficit sparing the semantic component, whereas Wernicke's aphasia is said to be associated with a deficit of the semantic component sparing syntax (cf. Marin, Saffran, and Schwartz 1976). The double dissociation in the deficits observed in these two aphasic syndromes has been regarded as evidence for the view that the language faculty consists of two autonomous semantic and syntax modules that can, hence, be selectively affected in language disorders. ${ }^{4}$

Moreover, data from language impaired speakers can also serve to evaluate competing linguistic theories. Out of a set of competing theories the one that best allows to capture the observed language deficits, i.e. the theory that allows to represent preserved and impaired structures or functions as natural classes within the theory, has to be preferred since it has the property of breakdown compatibility (Grodzinsky 1990). Grodzinsky argues that besides the criteria of learnability and parsability, breakdown compatibility constitutes a third criterion for evaluating the scientific quality of a theory.

With respect to exploring the human language faculty, a further goal of investigating language disorders is to find out about the localisation of specific grammatical capacities in the human brain. By exploring the correlations between specific language deficits and the localisation of the brain lesions causing these deficits, the hope is to identify those brain areas that critically subserve a specific grammatical structure or operation. Whereas modern imaging techniques such as PET and FMRT indicate all brain areas that are activated during a given cognitive operation, only lesion studies allow for identifying those brain areas whose intact functioning is in fact critical for performing this cognitive operation. A lesion of such a brain area should necessarily result in a deficit of the cognitive operation that critically depends on the normal functioning of this brain area (Rugg 1999).

Summarising, the linguistic investigation of language deficits offers a fascinating route to find out about the nature, the localisation and the functioning of the human language faculty. Also the study of language deficits has been considered to serve as "a natural laboratory in which linguistic theories may be tested" (Levi and Kavé 1999: 138). Nevertheless, despite this potential, researchers have been very careful to advance theoretical claims on the basis of data of language impairments (but cf. page 20 and notes 8 and 10). Rather than making use of impairment data to advance syntactic theory, syntactic theories have been used to explain impairment data.

\subsection{What are typical symptoms of syntactic deficits?}

A deficit affecting syntactic competence or the processing of syntactic structure in language performance has to be observable in language production and/or language comprehension. Whether or not a language impaired individual displays a syntactic deficit in language comprehension can only be revealed by explicitly testing an individual's language comprehension capacities. In contrast, symptoms suggestive of a syntactic deficit can in general quite readily be observed in the spontaneous-speech production of affected individuals and often require no explicit testing. Consequently, much research on syntactic deficits has concentrated on the signs of syntactic deficits that are observable in spontaneous-speech production.

\subsubsection{Typical signs indicative of syntactic deficits in spontaneous-speech production}

While deficit symptoms in spontaneous speech may vary between different language disorders, research has revealed a surprising correspondence of such symptoms across different language disorders. Among the core symptoms indicative of syntactic deficits in spontaneousspeech production are the following: 
- Problems with bound inflectional morphology are a core symptom in many language disorders (Penke 2008). Depending on the morphological system of the respective language, such problems might lead to the omission or substitution of inflectional markers. Omissions of inflectional markers only occur if the remaining word stem is a possible word in the respective language (such as book in two book or kiss in she kiss) (Grodzinsky 1984). Where the omission does not result in a possible word (e.g. Italian *libr- instead of libri $i_{[P L]}$, Russian *knig- instead of $\left.k^{*}{ }^{*} i_{[P L]}\right)$, inflectional markers are substituted - often by unmarked forms such as the infinitive or past participle in verbs and the nominative in nouns. Omissions and/or substitutions of bound inflectional morphology are a characteristic symptom of agrammatic Broca's aphasia (Menn and Obler 1990a) and Specific Language Impairment (Levy and Kavé 1999) across languages. Similar problems have, however, also been observed in Autism (Tager-Flusberg 2002), in Down syndrome (Laws and Bishop 2003), in children who have undergone hemispherectomy (Curtiss and Schaeffer 2005), and in Wernicke's aphasia (Kolk and Heeschen 1992) (see Penke 2008 for overview on inflectional disorders).

- Free function words such as determiners, auxiliaries, or conjunctions are often omitted. Such omissions characterise agrammatic Broca's aphasia (Menn and Obler 1990a), but similar omissions are also found in Specific Language Impairment (Leonard 1995), Down syndrome (Eadie et al. 2002), Autism (Bartolucci, Pierce, and Streiner 1980), and in children who have undergone hemispherectomy (Curtiss and Schaeffer 2005).

- Sentence length is reduced in language disorders such as agrammatic Broca's aphasia (Menn and Obler 1990a), Specific Language Impairment, Autism, and Down syndrome (Tager-Flusberg et al. 1990). Especially in Broca's aphasia spontaneous speech is often reduced to one- or two-word utterances which only include the central open class words of the intended utterance. Wernicke's aphasia is, in contrast, characterised by an increase in sentence length due to the wild-running concatenation of sentence constituents and formulas that often results in long utterances devoid of meaning (cf. Edwards 2005).

- The produced utterances are of reduced syntactic complexity. In languages which allow for different word orderings, canonical word ordering is preferred to other word order patterns. Thus, in German the canonical SVO order is preferred to the likewise grammatical OVS or XVS orderings in main clauses. Subordinate clauses are rarely produced in spontaneous speech, as are wh-questions or passives. This has been observed for agrammatic Broca's aphasia (Menn and Obler 1990a; Bates et al. 1988), Wernicke's aphasia (Niemi and Laine 1997; Bates et al. 1988), for Specific Language Impairment (Hamann 2006, Hamann et al. 2007), Down syndrome (Rondal and Comblain 1996), and for children who have undergone hemispherectomy (Curtiss and Schaeffer 2005).

- In languages with overt obligatory verb movement for finite verbs, verbs might appear in a non-finite form in main clauses and do not undergo verb movement. These structures are called root-clause infinitives. They regularly occur in agrammatic Broca's aphasia (Kolk and Heeschen 1992) and in Specific Language Impairment (Rice and Wexler 1996).

Whereas there is general agreement on the deficit symptoms that occur in spontaneous speech production of a given language disorder, the relevance of deficit symptoms that have been identified in especially designed language experiments tailored by individual researchers to test a specific syntactic-deficit account regarding a specific language disorder are often controversially discussed among researchers. Such controversies might centre on the issue whether the observed deficits are characteristic symptoms of a specific syntactic language 
disorder that can be observed in every affected individual, or whether the experimental set-up contains flaws in procedure, experimental material or subject choice that render the data invalid.

Nevertheless, carefully designed experiments that allow for a systematic collection of relevant data with respect to a critical syntactic construction are necessary to further our understanding of language disorders. A well-known limitation of spontaneous-speech data is that more complex syntactic structures are only rarely produced. However, absence of evidence is not necessarily evidence of absence. That is, we simply cannot know whether a specific form or construction is missing because the individual can no longer produce it due to the language deficit, or whether it is not showing up by coincidence, for example, because we just did not look at a large enough data set. A systematic collection of relevant data in an experiment allows for disentangling this issue. Indeed, comparisons between elicited and spontaneous speech data have shown that constructions or forms whose production poses a problem for a language impaired speaker are often avoided in spontaneous-speech production and only show up when the context provided in the experimental task requires the formation of a specific form or construction (Kolk and Heeschen 1992; Penke 2006).

A further advantage of experimental studies is that the researcher can control for all sorts of confounding factors that might influence the individual's language behaviour, such as, for instance, word-form frequency and the length of sentences. Thus, language impaired speakers might experience problems with agreement or case inflection, but correctly use inflected suppletive forms that have a very high frequency of occurrence (Penke 1998: 200- 202, 211212). Also, a syntactic construction might pose problems for a subject simply because it contains more words to produce, parse or remember than another less affected construction. In a carefully designed experiment, such potentially influencing factors are controlled for. On the other hand, the control over the experimental material and the reaction of the tested individual is obtained at a price, namely the (sometimes highly) artificial situation which might also influence the individual's language behaviour (e.g. Heeschen and Schegloff 2003). Thus, experimental data has to deal with the critique that observed deficits are due to the unnaturalness of the situation and might just constitute an artefact caused by the chosen experimental design. Hence, it is important to adopt different methodologies in experimental testing.

\subsubsection{Typical signs indicative of syntactic deficits in language comprehension}

While symptoms indicative of syntactic deficits are readily observable in spontaneous-speech production, syntactic deficits in language comprehension are hidden from simple observation and can only be revealed by explicit testing. As mentioned above, experimental data are susceptible to criticism targeting the experimental set-up. Thus, it can be argued that the number of items is too small to obtain reliable results or that confounding factors have not been sufficiently controlled for. Moreover, whereas spontaneous-speech symptoms are generally seen as facts which are rarely disputed, the issue of syntactic comprehension deficits targets a theoretical issue related to the nature of the language faculty and the presence or absence of comprehension deficits is, therefore, hotly disputed. The controversy is on the following issue: Chomsky's generative approach to the human language faculty predicts that the language organ underlying this faculty should be affected by brain lesions leading to language disorders. A deficit impairing the syntactic competence of an individual should affect language production and language comprehension in parallel (Weigl and Bierwisch 1970), since the human abstract grammatical competence is put to use in both, language production and comprehension (i.e. in performance) (Chomsky 1980). Consequently, researchers who assume that syntactic disorders are due to a deficit in syntactic competence try to provide evidence for a deficit that affects all facets of language performance (language production, comprehension and judgement) in 
parallel. In contrast, researchers who assume that there is no such thing as syntactic competence, that syntactic disorders are due to modality specific processing disorders or that syntactic competence is basically intact in language disorders aim to show that language comprehension is not affected or, at least, not affected in parallel to language production.

A classic design in testing language comprehension capacities is the sentence-picture matching task where the subject has to match a given sentence such as The boy is kissed by the girl to one of two pictures, one depicting the action described in the sentence (e.g. girl kissing boy), the other depicting the reverse action (e.g. boy kissing girl). Studies on language comprehension capabilities adopting this or related designs have revealed that in languages with a canonical SVO word order, most language-impaired individuals display a better understanding of sentences with this canonical word order compared to sentences with a noncanonical word order such as object-clefts (it is the boy who the girl kissed), object relatives (the boy who the girl kissed...), passives (the boy is kissed by the girl), or object topicalisations where the object has moved out of its base-generated position and precedes the subject. The typical error in the comprehension of these constructions is that the first noun phrase occurring in the sentence is interpreted as the AGENT of the action. Whereas this does not lead to comprehension problems when the first noun phrase is indeed the AGENT, such as in active SVO sentences (the girl kissed the boy), subject cleft constructions (it is the girl that kissed the boy), or subject relative clauses (the girl who kissed the boy ...), problems arise when the first noun phrase is the THEME of the action. Then, for example, a passive clause such as The boy is kissed by the girl might be interpreted as boy kissed girl.

The English passive construction is certainly the best investigated construction in language comprehension studies. A good understanding of active sentences compared to an impaired understanding of passive sentences (e.g. The boy is kissed by the girl = boy kissed girl) has been observed in Broca's aphasia (Grodzinsky 2000) and Wernicke's aphasia (Bastiaanse and Edwards 2004), in dementia of the Alzheimer type (Grober and Bang 1995), in Autism (TagerFlusberg 1981), Specific Language Impairment (Van der Lely 1996), Down syndrome (Ring and Clahsen 2005), in children with early unilateral focal brain lesions (Dick et al. 2004), and in children who had to undergo a resection of the left brain hemisphere (Dennis and Whitaker 1976)..$^{5}$

These findings have, however, not gone unchallenged and heated discussions have ignited on the comprehension problems in agrammatic Broca's aphasia. For one, a subject-object asymmetry resulting from the strategy to regard the first noun phrase as AGENT of the action is also regularly observed in control subjects who do not suffer from any language impairment. Thus, this asymmetry and the underlying comprehension strategy do not seem to be specific for syntactic deficits in language impaired speakers. Also, there has been a fierce controversy on the issue how to deal with individual language impaired speakers who do not display the described comprehension problem. A comparison of language comprehension abilities in a group of unimpaired control subjects and a group of language impaired speakers, will typically yield that the group of language impaired speakers does significantly worse in the comprehension of non-canonical structures (such as passives) compared to the group of unimpaired control subjects. Whereas this finding will hold for the group, there might be some language impaired individuals who will perform within the range of the unimpaired control subjects. A number of case studies of agrammatic Broca's aphasics who do not display problems in understanding non-canonical sentences have indeed been published (cf. Berndt, Mitchum, and Haendiges 1996 for overview). One group of researchers considers these studies to provide evidence for the claim that language comprehension problems are no characteristic sign of Broca's aphasia and, therefore, do not have to be captured in deficit theories accounting 
for this disorder. The other group, however, argues that these cases are to be expected in a normally-distributed group of language impaired subjects and constitute no counter-cases for the claim that the described language comprehension deficit is a characteristic symptom in Broca's aphasia (cf. Grodzinsky 2000; Drai and Grodzinsky 2006 and the subsequent discussions on methodological considerations how to compare and evaluate group differences).

Besides the above described problems in understanding non-canonical sentence structures, comprehension problems have been found in the area of binding. Interestingly, however, it seems as if problems in this area might be specific to different language impairments. Children with Specific Language Impairment have been found to sometimes give an anaphorical reading to pronouns. Thus, they will choose a picture showing that 'Mowgli tickles himself' as adequate depiction of the sentence Mowgli tickles him (Van der Lely and Stollwerck 1997). A similar behaviour has been observed in individuals with Broca's aphasia (Grodzinsky et al. 1993). Wernicke's aphasics, in contrast, display a general disruption in understanding pronouns and thus, will also judge that a sentence like Mowgli tickles him does not depict a picture where Mowgli tickles Baloo (Grodzinsky et al. 1993). A different picture is again observed in individuals with Down syndrome. These individuals experience problems in understanding reflexive pronouns and will accept a picture showing that 'Mowgli tickles him' as illustration for the sentence Mowgli tickles himself (Ring and Clahsen 2005). In contrast to all these groups, individuals with Williams syndrome have been found to be unimpaired with respect to the binding of anaphors (Clahsen and Almazan 1998).

\section{Accounting for syntactic deficits}

Over the last 30 years a multitude of deficit approaches have been advocated to capture the above described symptoms as resulting from syntactic deficits. Such attempts range from deficit theories which assume rather global deficits encompassing all of syntax, to approaches which presuppose rather subtle deficits affecting only particular syntactic structures, processes, or features. Most of these attempts have been formulated within the framework of Generative Grammar and here within the theories of Government and Binding (Chomsky 1981) and the Minimalist Program (Chomsky 1995, 2000).

\subsection{Global syntactic deficit accounts}

The first syntactic deficit theories proposed to account for language impairments found in agrammatic Broca's aphasia date from the late 1970s and the early 1980s. The framework of Generative Grammar was still in its youth by then and the focus of research on language disorders was in finding evidence for the assumption that the human language faculty consists of autonomous modules such as a syntax module (see section 1.1.). Given this research agenda, researchers focussed on the issue whether Broca's aphasia could be described as a language disorder that selectively affected the syntax component, sparing the semantic component (e.g. Caramazza and Berndt 1978), and whether this disorder was due to an impairment in syntactic competence that affected language production and comprehension in parallel (e.g. Heilman and Scholes 1970; Caramazza and Zurif 1976) (see section 1.2.2.).

Syntactic deficit approaches to agrammatic Broca's aphasia assumed rather global deficits encompassing all syntactic capacities. An example for these approaches is the Lexical-Node Hypothesis suggested by Caplan (1985). According to this approach, agrammatic Broca's aphasics are not able to construct syntactic phrase structures. They only have access to lexical categories such as verbs and nouns and to the information that is stored with these lexical elements in the mental lexicon. Besides semantic information this includes syntactic information on word class, subcategorisation frames and thematic roles. Since the capacity to build up 
hierarchically ordered syntactic structures is lost in agrammatic Broca's aphasia, the main lexical elements are linearly ordered. Thematic roles such as AGENT or THEME are assigned to linear positions in these word strings: AGENT is assigned to the noun in front of the verb, THEME is assigned to the noun coming after the verb (cf. fig.1). The incapacity to build-up phrase-structure representations will also mean that syntactic operations or relations which build on phrase structures, such as movement operations or the establishment of syntactic relations between hierarchically ordered constituents, are no longer possible. With this account, Caplan tried to provide a unitary account for the omission of function words and inflections, for the preponderance of canonical sentence structures and for the comprehension problems that result when sentences with non-canonical ordering are presented.

linear ordering

Figure 1: Sentence representations according to a global syntactic deficit account

\subsection{Deficits in structure building}

With time and concurring with the development of generative syntax, more fine-grained accounts of syntactic deficits were developed that did not longer assume a global deficit affecting all syntactic operations and relations. Instead, approaches were proposed that suggested more specific deficits in the build up of syntactic phrase structure.

\subsubsection{Surface-structure-deficit accounts}

With the elaboration of the theory of Government and Binding (Chomsky 1981), the distinction between a thematic $D(e e p)$-structure and a $S$ (urface)-structure presented itself as an obvious candidate in accounting for the syntactic deficits observed in Broca's aphasia. D(eep)-structure is the level that encodes the lexical properties of the sentence elements and the thematic relations that hold between these elements. Surface properties of the sentence such as the ordering of sentence elements and the inflection of these elements are reflected by S(urface)structure which is derived from $\mathrm{D}(\mathrm{eep})$-structure by movement transformations. Functional heads and their projections are central in deriving S(urface)-structure. Functional heads host inflectional morphology such as agreement, tense, and case markings that have to be associated with lexical elements such as verbs and nouns. Functional elements such as complementisers and auxiliaries are base-generated in functional heads such as C, T, and AGR and functional heads and their specifiers offer landing sites for constituents that move out of their base-generated D-structure positions (Haegeman 1991).

As presented above (see section 1.2.1.), the omission of functional elements (such as articles, complementisers, and auxiliaries) and substitutions respectively omissions of inflectional morphology are the core symptoms of agrammatic language production. In contrast, lexical information (e.g. word class and subcategorisation information) and the establishment of semantic relations between lexical elements seem relatively unimpaired in Broca's aphasia. This dissociation led researchers to hypothesise that agrammatic Broca's aphasia might be due to an impairment in deriving S(urface)-structure representations - an impairment that ultimately results from a deficit in projecting functional heads (e.g. De Bleser and Bayer 1991; Ouhalla 1993). According to Ouhalla (1993) for instance, agrammatic individuals no longer have access 
to the inventory of functional categories that is part of Universal Grammar and, hence, can no longer project these functional heads into syntax. While the derivation of S-structure is impaired according to these approaches, all information that comes from the lexicon is unaffected and can be projected into syntax. Thus, the build-up of lexical projections such as VPs, NPs, and PPs according to the $X^{\prime}$-scheme is still possible and the argument structure as well as the thematic structure of lexical heads can be expressed. In consequence then, the build-up of syntactic structure is reduced to the D-structural level in these approaches (cf. figure 2). All syntactic operations that require functional heads and their projections at the S-structural level are, however, impaired.

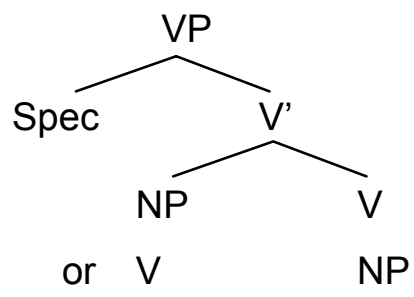

Figure 2: Sentence representations according to a S(urface)-structure-deficit account

While S(urface)-structure-deficit approaches differ with respect to whether or not inflected forms can be extracted from the lexicon, the deficit in building-up S(urface)-structure representations should in any case affect movement transformations. Movement transformations require a landing site for the moved constituent. These landing sites are provided by functional heads and their specifier positions. If functional heads are no longer projected, systematic movement transformations should not be observable in agrammatic speech production. Obligatory verb movement has provided an excellent test case for this prediction. Consider German, a V2 language where the finite verb has to move to the $C$ position, the second structural position, in main clauses. Non-finite verbs, in contrast, remain clause-finally in the $\mathrm{V}$ position of the headfinal German VP (cf. figure 3).

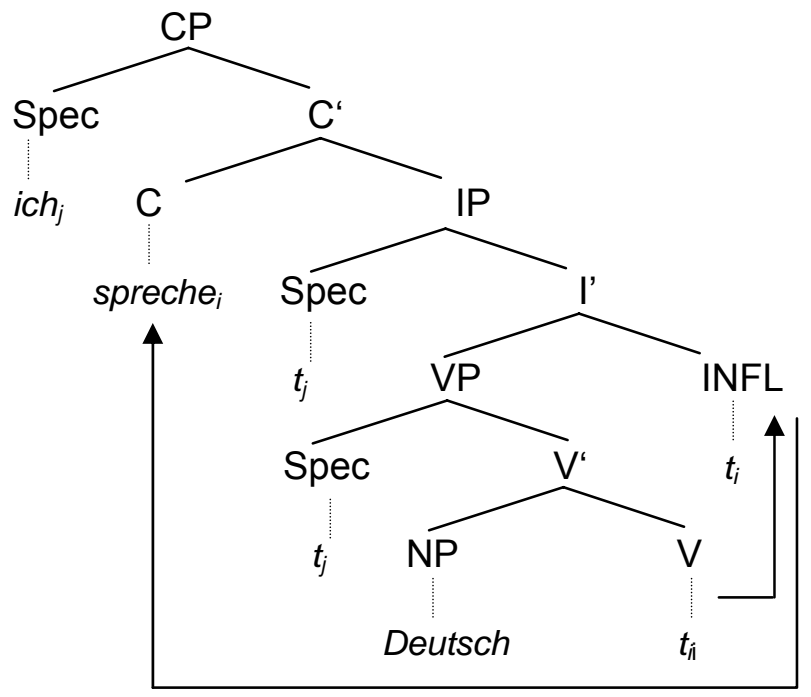

Figure 3: V2 movement in German ${ }^{6}$

An inability to build-up S(urface)-structure and to project the functional head $\mathrm{C}$ should necessarily affect the obligatory V2 movement of finite verbs in German main clauses. In 
consequence, a systematic V2 placement of finite verbs in main clauses is not expected to occur in the language production of German agrammatic Broca's aphasics. However, an investigation of spontaneous and elicited speech produced by four German agrammatic aphasics revealed that out of 615 finite verbs for which verb placement could be unambiguously determined, 607 (98.7\%) were correctly placed in V2 position (Penke 2001). Only 2\% were incorrectly placed clause-finally. In contrast to the placement of finite verbs, $97.8 \%$ of the 182 non-finite verbs produced by these subjects were left clause-finally in the VP. These data indicate that the V2 placement of finite verbs and the resulting asymmetry in the positioning of finite and non-finite verbs in German main clauses are retained in German individuals with Broca's aphasia. Since V2 movement requires a functional projection outside VP, the data from German, as well as similar data from Dutch, French, and Italian Broca's aphasics (Kolk and Heeschen 1992; Lonzi and Luzzatti 1993), provide strong evidence against deficit approaches which assume that functional categories can no longer be projected in agrammatic aphasia.

\subsubsection{Deficits affecting the build-up of specific functional projections:}

As indicated above, deficit approaches assuming that all functional heads and their projections are impaired in a language disorder such as agrammatic Broca's aphasia seem to posit too much a deficit. As the data on verb movement in Broca's aphasia indicate, not all functional heads are missing from agrammatic $S$ (urface)-structure representations. Research consequently focussed on the syntactic functions specific functional categories have, exploring whether or not these particular functions are retained or impaired in language disordered subjects. These endeavours were furthered by developments in syntactic theory such as the Split-INFL Hypothesis (Pollock 1989) which proposed that the functional category INFL should be divided up into two independent functional categories: T for tense marking and AGR for establishing subject-verb agreement. This proposal and subsequent related work increased the number of potential functional heads to investigate in language disorders. As result of this research agenda, a number of dissociations have been reported in the literature on syntactic deficits, indicating that while syntactic functions related to specific functional categories are impaired, syntactic functions related to other functional categories are retained in particular language disorders.

Consider for example the Tree-Pruning Hypothesis advocated by Friedmann and Grodzinsky (1997) which claims that syntactic structures in agrammatic aphasics are "pruned" at the tense node. The account was proposed on the basis of data from a Hebrew-speaking agrammatic aphasic subject who displayed a strong dissociation within verbal morphology. While this subject had a good command of agreement inflection, she markedly failed in producing verb forms correctly inflected for tense. Moreover, this subject could not produce embedded sentences and wh-questions, omitting the sentence-initial complementiser or whphrase most of the time. In accounting for this data, Friedmann and Grodzinsky made use of the Split-INFL Hypothesis and assumed an ordering of functional heads according to which $C$ is higher than $T$ which is higher than AGR. Given this ordering, the observed deficit can be captured by the assumption that all functional heads from $T$ upwards can no longer be projected in agrammatic representations, thus accounting for retained agreement inflection in this subject. The Tree-Pruning Hypothesis implies that all syntactic operations that necessarily rely on the functional projection TP and on functional projections higher up the syntactic tree, such as CP, can no longer be performed in agrammatic subjects. 


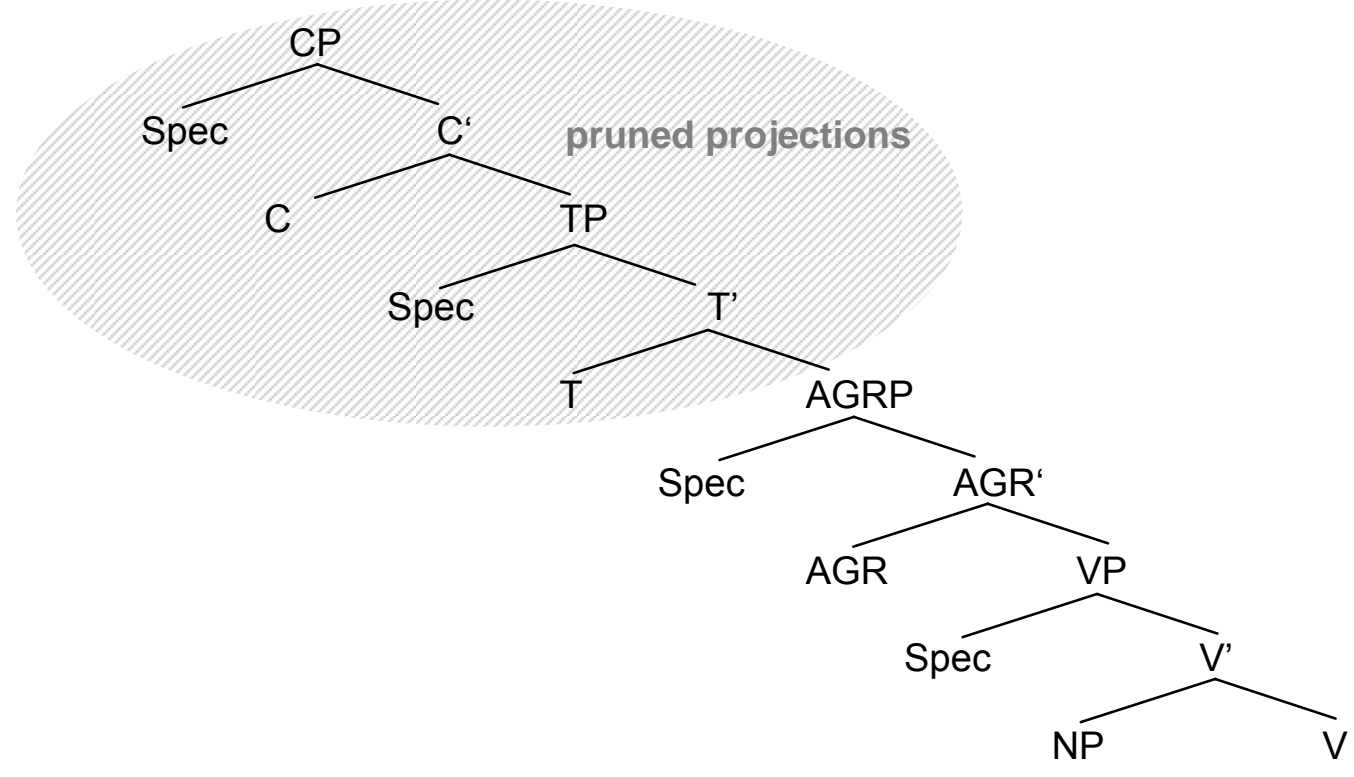

Figure 4: A pruned syntactic representation according to the Tree-Pruning Hypothesis

To deal with the finding that deficit accounts which assume all functional categories to be impaired are too broad, Friedmann and Grodzinsky (1997) and other researchers have also suggested to relate the severity of the syntactic deficit to the type and number of functional heads affected. The more severe the agrammatic deficit, the fewer functional projections can be built up (Friedmann and Grodzinsky 1997) or generated by the syntactic operation 'merge' (Hagiwara 1995). Whereas a mild deficit might only affect the CP layer in phrase-structure representations, a severe deficit might affect the build-up of all functional projections down to the VP layer.

The integration of this idea into accounts such as the Tree-Pruning Hypothesis is certainly interesting since language deficits are gradable and come in different levels of severity in different individuals. However, at present the suggestion to relate the severity of the disorder to the amount of functional heads that can be projected by language impaired individuals suffers from a lack of independent criteria to indisputably establish the severity of the disorder in a given subject. Without independent criteria to establish the severity of a language deficit circular arguments are likely to arise. Hence, a deficit in producing a language structure X (e.g. V2 movement) is taken as evidence for a severe disorder. A severe disorder is claimed to lead to a pruning of functional nodes high-up in the syntactic tree (e.g. CP). Evidence that the respective functional layer (CP) is indeed pruned is then claimed to come from the observation that structure $X$ (e.g. V2 movement) is impaired. As long as independent criteria are missing, potential counter-evidence to these deficit-approaches - such as the data on German verb movement which indicate that the functional head $\mathrm{C}$ can still be projected in agrammatic aphasic subjects - is discarded by claiming that these data come from individuals who are not impaired severely enough to display the suggested deficit. In practise, this renders the falsification of gradable deficit theories quite impossible.

Despite empirical controversies on the issue whether or not the Tree-Pruning Hypothesis adequately captures the deficits observed in agrammatic Broca's aphasia across languages, the Tree-Pruning Hypothesis has also been challenged on theoretical grounds. For one, it has been argued that the suggested order of functional categories that places the T node above the AGR node does not hold across languages. In German, for instance, the AGR node is placed above 
the T node reflecting affix-order in German verbs (Baker's 1985 Mirror Principle, cf. Penke 2000). A pruning of the syntactic tree above the T node would, hence, lead to an inability to project any functional categories in German - a prediction which is not borne out since agreement inflection (involving the AGR node) and V2 movement (involving the C node) are largely intact in German agrammatic aphasics (Penke 2000). Also, in more recent developments of the Minimalist Program tense and agreement are no longer treated as two independent functional categories (Chomsky 2000), but agreement is an operation checking uninterpretable features of $\mathrm{T}$. Without an independent functional category AGR, however, the dissociation between retained agreement inflection and impaired tense inflection observed by Friedmann and Grodzinsky (1997) can no longer be captured in the Tree-Pruning account, since agreement, like tense inflection, would involve the pruned T node (cf. Wenzlaff \& Clahsen 2004).

\subsection{Deficits with the feature specification of functional heads}

A different type of deficit theories assumes that while the build-up of syntactic structure via the projection of functional heads is intact, the syntactic features that are hosted by these functional categories are underspecified. This type of approach goes back to an account on agrammatic language production proposed by Grodzinsky (1984).

Due to the Anglo-American dominance in modern generative linguistic research, most research on language impairments up to the early 1980s was concerned with the deficits that were observed in English-speaking individuals. Since the omission of inflectional affixes is a core symptom of English speakers with Broca's aphasia, omissions of inflectional markers were seen as characteristic sign of this disorder across languages. The assumption that across languages bound and free functional elements (inflectional markers and function words) are omitted in Broca's aphasia seemed to suggest that the build-up of syntactic structure, specifically the build-up of functional projections, was impaired in agrammatic individuals (see sections 2.2.1.-2.2.2.). In comparing deficits associated with agrammatic Broca's aphasia across languages, Grodzinsky (1984), however, observed that omissions of inflectional markers only occur in agrammatic speech production if the remaining word stem is a possible word in the respective language, as is the case in English. In languages such as Hebrew, Russian, or Italian, where the omission of inflectional markers results in stems which cannot surface as possible words in these languages (Italian *libr- instead of libri, Russian *knig- instead of knigi), omission errors do not occur. Rather, in these languages inflectional markers are often substituted by other markers in the speech of agrammatic aphasic individuals, resulting in inflectional errors.

Such inflectional errors are problematic for deficit accounts which assume that all functional projections are missing in syntactic representations of agrammatic individuals. According to the Government and Binding theory, the standard syntactic framework at this time, inflectional morphology is hosted by functional heads. Hence, the presence of inflectional markers, even incorrect ones, indicated that syntactic representations contain functional heads that dominate these inflectional markers. Therefore, Grodzinsky suggested that the build-up of S(urface)structure representations itself is not impaired. However, functional heads are underspecified, since the specific feature values of the syntactic features dominated by functional heads are deleted in agrammatic syntactic representations (cf. fig. 5). As a consequence, free standing functional elements such as articles or auxiliaries are omitted in agrammatic language production. In addition, the deletion of specific feature values results in omissions of bound inflectional morphology if the omission of an inflectional marker leads to a possible surface word in the respective language. In languages where omissions are not an option, any feature value, 
matching or not, is selected for an underspecified syntactic feature and, hence, any possible inflectional form can be produced - the correct one or an incorrect one. Given these considerations, a sentence such as the boy is kissing the girl would consequently be produced as boy kiss girl by English-speaking agrammatic subjects.

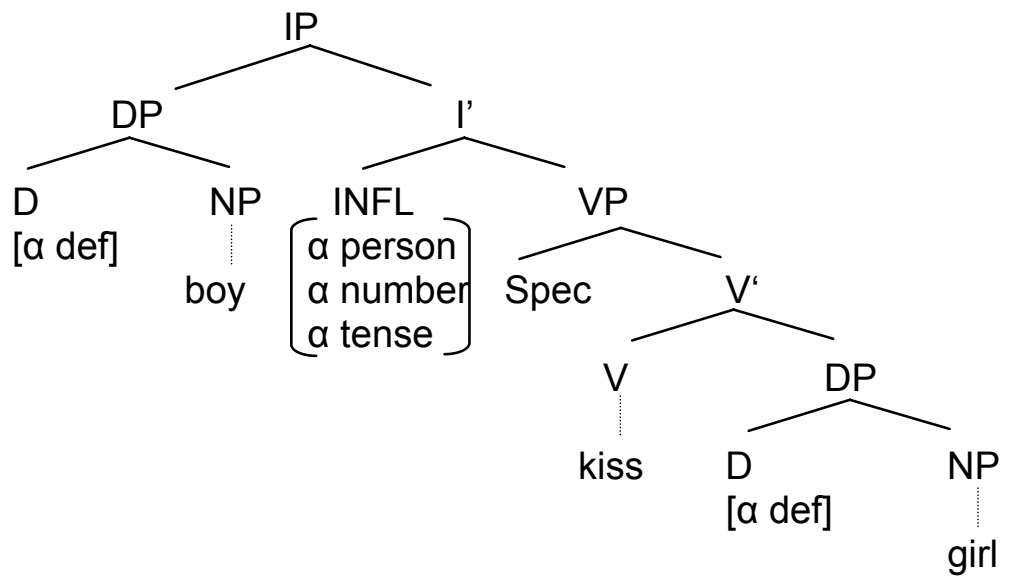

Figure 5: Syntactic S(urface)-structure representation with underspecified functional heads, resulting in the 'agrammatic' sentence boy kiss girl

Grodzinsky's finding that omissions of inflectional elements will only occur where licensed by the grammar of a language constitutes an important generalisation on inflectional deficits in language disorders. However, his proposal that all functional heads are underspecified turned out to be too strong. Consider for instance subject-verb agreement inflection in German Broca's aphasics. According to Grodzinsky's account the agreement features dominated by the functional head INFL are underspecified. Consequently, agreement affixes should be omitted since this is a morphologically legitimate option in German. In contrast to this prediction, subject-verb inflection is basically intact in German Broca's aphasics. An analysis of subject verb agreement inflection in spontaneous speech production (Penke 1998: 191) and several elicitation experiments (Penke 1998: 192-195; Janssen and Penke 2002) conducted with a total of 14 German agrammatic aphasics revealed that all tested individuals were able to systematically mark agreement in German (individual correctness scores ranging from $71 \%$ to $100 \%$, mean $92 \%$ ).

Nevertheless, Grodzinsky's suggestion that syntactic deficits could be due to underspecified feature values of functional heads has inspired researchers in the field, a reason certainly being the advent of the Minimalist Program (Chomsky 1995, 2000) in which syntactic features play a central role in the derivation of syntactic structure. In the Minimalist Program, functional heads dominate bundles of syntactic features that have to be checked against the inflectional endings of a form generated in the lexicon. Feature checking will eliminate the abstract features dominated by functional heads. To enable feature checking the inflected form has to move into a checking domain of the functional head hosting the respective syntactic feature. If the syntactic features realized by an inflected form are compatible with the syntactic features dominated by a functional head, the syntactic features headed by this functional category can be checked off and will be eliminated from the syntactic representation. The Minimalist Program draws a distinction between different types of features. Strong features force overt movement, i.e. feature checking has to occur prior to spell-out. Weak features are checked after spell-out. Their movement into the checking domain therefore is covert, i.e. not visible on the surface. Another distinction is drawn between interpretable and uninterpretable 
features. Interpretable features can be interpreted at the logical form level, i.e. they are relevant to the semantic interpretation of an expression. Uninterpretable features, in contrast, cannot be interpreted at this level and need to be checked off before the logical form level, lest the derivation crashes at this level.

The issue whether syntactic impairments can be related to deficits causing the underspecification of specific features while sparing others has inspired some research during the last 15 years. Rice and Wexler (1996) adopted this line of explanation to account for the observation that children with Specific Language Impairment display a protracted period where they optionally produce root infinitives instead of main clauses with finite verbs (cf. section 1.2.1.). According to the Tense-Omission Model (Wexler 1994) an optional infinitive results when the child leaves the tense feature of the functional category INFL underspecified. In this case, the verb displays non-finite inflection and does not undergo verb movement. In children with Specific Language Impairment the time period where the tense feature may be left underspecified is said to be extended (Extended Optional Infinitive Hypothesis). Whereas the tense feature may be unspecified in syntactic representations of children with Specific Language Impairment, all other functional categories, features, or syntactic operations are said to be intact. This claim is, however, disputed on the basis of data indicating that subject-verb agreement inflection is impaired in Specific Language Impairment although the agreement features should be unaffected according to the Extended Optional Infinitive Hypothesis (cf. Clahsen, Bartke, and Göllner 1997). ${ }^{7}$

Based on the observation that verbal agreement inflection is impaired in German children with Specific Language Impairment, whereas tense marking is significantly better retained, Clahsen, Bartke, and Gölner (1997), have proposed that Specific Language Impairment is due to a deficit particularly affecting the uninterpretable phi-features of verbs, i.e. the verb's agreement features. ${ }^{8} \mathrm{~A}$ reverse deficit affecting interpretable tense features has been claimed to account for the observation that tense inflection is more impaired than verbal agreement inflection in German agrammatic Broca's aphasics (Wenzlaff and Clahsen 2004). Note, however, that the language deficits in Specific Language Impairment and agrammatic aphasia are not as selective as suggested above. Whereas Clahsen, Bartke, and Göllner (1997) state that only agreement but not tense inflection is impaired in Specific Language Impairment, a number of studies have found tense inflection to be severely affected in English children with Specific Language Impairment (e.g. Rice and Wexler 1996; van der Lely and Ullman 2001). Conversely, deficits with agreement inflection are typically observed in English-speaking Broca's aphasics (e.g. Goodglass et al. 1993; Faroqi-Shah and Thompson 2004), contrary to the claim of Wenzlaff and Clahsen (2004).

Rather, the findings suggest that deficits with respect to inflectional morphology vary across languages. In English, an analytic language with a largely reduced inflectional component, inflectional markers - be it tense or agreement markers - tend to be omitted, resulting in inflectional errors. In languages where inflectional systems are more elaborate and express more syntactic information (such as in Finnish, German, Italian, Polish, or Spanish) omission rates of inflectional markers are markedly lower compared to English (e.g. Bates et al. 1987; Dromi et al. 1999). Thus, whereas for instance the English $3^{\text {rd }}$ person singular marker $-s$ in English is omitted in about half of the obligatory contexts for this marker by children with Specific Language Impairment (Clahsen, Bartke, and Göllner 1997) and adult Broca's aphasics (Goodglass et al. 1993), omission rates for subject-verb agreement inflection in German speaking subjects with these disorders are considerably lower. Clahsen, Bartke, and Göllner (1997) report an omission rate of $20 \%$ for German children with Specific Language Impairment. The five agrammatic aphasic subjects investigated by Penke (1998: 191) omit agreement 
affixes in only 15 out of 914 cases $(1.6 \%)$ in spontaneous speech production. Such variations across languages suggest that language-specific factors related to the complexity and importance of morphological inflectional systems critically affect which inflectional errors will occur in language impaired speakers (see Penke 2008 for discussion). Note that languagespecific variations in inflectional errors are problematic for deficit theories supposing selective deficits with interpretable or uninterpretable features. As the categorisation as uninterpretable or interpretable feature holds for all languages, similar deficits with tense or agreement inflection should be observed across languages. At present, the evidence for deficit accounts, which suggest that the impairments observed in language disorders such as Specific Language Impairment and Agrammatism are selective to specific features while sparing others is inconclusive.

\subsection{Deficits with movement}

As presented in section 1.2.2., language impaired individuals often show a better understanding of sentences with a canonical SVO order of sentence constituents (the girl kissed the boy) compared to sentences with a non-canonical word order such as object-clefts (it is the boy who the girl kissed), object relatives (the boy who the girl kissed...), passives (the boy was kissed by the girl), or object topicalisations. What is common to these sentence structures is that in all these cases the object has moved out of its base-generated position and now precedes the subject. Grodzinsky has, therefore, suggested that it is the movement of the object that causes comprehension deficits in Broca's aphasia (Grodzinsky 1984, 2000). Specifically, he proposed that the deficits in language comprehension are due to the fact that movement traces in syntactically derived sentences, such as passives or object extracted questions, are deleted from syntactic representations.

In the framework of Government and Binding (Chomsky 1981), S(urface)-structure is derived from $\mathrm{D}$ (eep)-structure by movement transformations. Every moved constituent leaves behind a trace. The moved constituent and its trace are connected via a syntactic chain. In case of moved noun phrases, the most important syntactic function of this chain is to transmit the theta role assigned by the verb to its arguments within VP to the moved constituent. The theta role is assigned within VP to the trace of the moved constituent. Via the syntactic chain this theta role is then indirectly assigned to the moved constituent co-indexed with this trace (cf. Haegeman 1991).

According to Grodzinsky's Trace-Deletion Hypothesis such traces are deleted from the syntactic representations of Broca's aphasics. Consider for instance a passive clause as in (1). In a passive clause, the object NP has to move out of VP to the subject position in SpecTP to receive case. It leaves behind a co-indexed trace in its base-position as internal argument of the verb kiss. The theta role THEME is assigned by the verb to this trace. Via the syntactic chain connecting the moved NP with its trace, this theta role is indirectly assigned to the moved NP, the derived subject of the sentence (cf. Haegeman 1991). The theta role AGENT is assigned by the preposition by. In an agrammatic representation, however, movement traces are deleted. Consequently, the theta role THEME cannot be assigned to the moved object NP via the disrupted chain. Since for interpretation every NP must have a theta role, the NP is now assigned a role by a non-linguistic linear default strategy. This default strategy assigns the AGENT role to the clause-initial NP. Since the preposition by also directly assigns the role AGENT to its complement NP, the thematic representation now contains two AGENT roles to choose from in interpretation: one assigned via the default strategy, the other directly assigned by the preposition. As a consequence, in a sentence-picture-matching task (cf. section 1.2.2.) the comprehension of such sentences will drop to chance level. 
(1)

unimpaired representation of passives

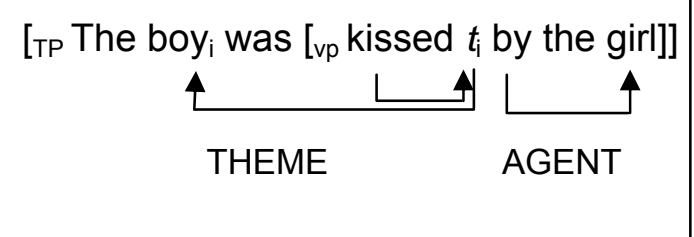

agrammatic representation

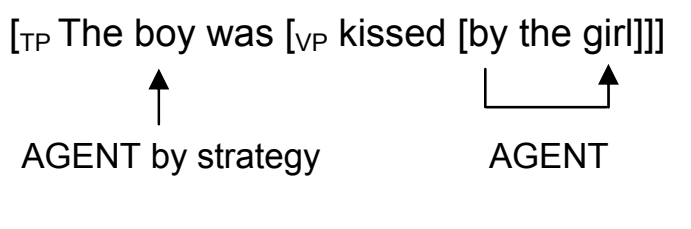

For active sentences such as in (2), in contrast, no problem arises in agrammatic comprehension, since both theta roles can be directly assigned: the THEME role is assigned by the verb to its internal argument, the AGENT role is directly assigned to the subject basegenerated in SpecTP. ${ }^{9}$

(2) unimpaired/agrammatic representation for active sentences

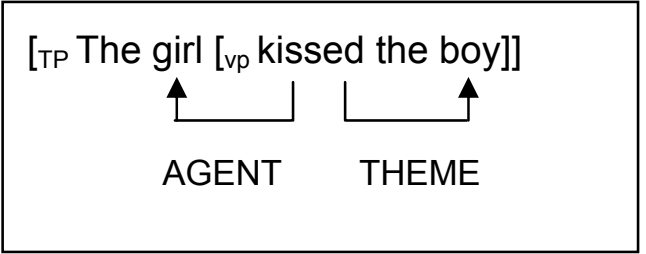

The Trace-Deletion Hypothesis has been the most influential deficit theory in the field to date. It has inspired a wealth of research aimed at testing and improving this account. A strength of this theory certainly lies in the fact, that it has been flexible enough to adapt to both, new findings on agrammatic comprehension and new developments in syntactic theory.

Originally, the Trace-Deletion account stated that all types of traces, including traces of moved heads, were deleted in agrammatic representations. Based on evidence that head chains are intact in agrammatic comprehension, the account was modified in the way that only traces in theta positions are said to be deleted in agrammatic representations (Grodzinsky 1995). A further modification was initiated by Hickok and Avrutin (1995) who tested the comprehension of wh-questions in Broca's aphasics. The Trace Deletion Hypothesis predicts that the comprehension of wh-object questions (which boy/who did the girl kiss?) should be at chance level in agrammatic aphasic subjects. The moved object-wh-phrase cannot receive a theta role via the disrupted chain and receives the AGENT role via the default strategy. Since the subject NP is also assigned the AGENT role (by the verb), the conflict between two AGENT roles should lead to chance performance in interpretation. Hickok and Avrutin, however, observed that their agrammatic subjects showed this behavioural pattern only for which $N$ object questions, but not for who object questions. This led to a revision of the Trace-Deletion Hypothesis according to which a default theta role is only assigned to a referential NP, i.e. a NP which is $\mathrm{D}$ (iscourse)-linked, as is the cases for which $N$ phrases but not for who phrases which are said to be quantifiers and thus not D-linked (Grodzinsky 1995).

The Trace-Deletion Hypothesis has not only had to adapt to new types of data, but it has also been affected by developments in syntactic theory, most notably by the VP-internal Subject Hypothesis (Koopman and Sportiche 1988) according to which the subject NP is not basegenerated in SpecTP but in SpecVP and subsequently moves to SpecTP to check its features, thus leaving a trace inside SpecVP. Integrating this assumption into the Trace-Deletion Hypothesis has turned out to be quite challenging. ${ }^{10}$ Consider first a simple active sentence such as (3). In the agrammatic representation of this sentence the AGENT role can no longer 
be assigned to the subject NP moved out of VP since the trace is deleted. Nevertheless, comprehension of such sentences is not affected in Broca's aphasics because the subject NP receives the AGENT role via the default strategy (cf. 3 ).

(3)

unimpaired representation of actives

[TP The girl $_{\mathrm{i}}\left[\mathrm{VP} t_{\mathrm{i}}\right.$ kissed the boy]]

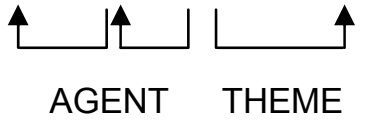

agrammatic representation

[TP The girl [vP kissed the boy]]

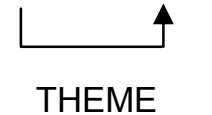

For other sentence constructions, however, the integration of the VP-Internal Subject Hypothesis into the Trace-Deletion Hypothesis has led to predictions that are not born out by the agrammatic comprehension data. Consider as an example wh-object questions such as (4). In a wh-object question neither the object NP nor the subject NP receive a theta role directly by the verb because both phrases are moved out of VP and their traces are deleted. The default strategy would then assign the AGENT role to the clause-initial NP, the object wh-pronoun. In consequence, wh-object questions should be consistently interpreted as wh-subject questions by agrammatic individuals and their performance in understanding such sentences should be worse than chance, a prediction which is, however, not born out by the data (cf. Hickok and Avrutin 1995, see Groszinsky 2000: 59 for suggestions how to solve this issue within the TraceDeletion Hypothesis). ${ }^{11}$

(4)

agrammatic representation

[CP who/which boy did $_{\text {TPP }}$ the girl ${ }_{\mathrm{i}}\left[\mathrm{vP} t_{\mathrm{i}}\right.$ kiss $\left.\left.\left.t_{\mathrm{j}}\right]\right]\right]$<smiles>[13CH3]</smiles>

AGENT by strategy

Deficit accounts related to the Trace-Deletion Hypothesis - which is explicitly restricted to capture only agrammatic language comprehension (cf. Grodzinsky 2000) - have meanwhile been adopted to capture similar problems in individuals with Wernicke's aphasia (Grodzinsky and Finkel 1998) and in children with Specific Language Impairment (Friedmann and Novogrodsky 2004) or hearing impairments (Friedmann and Szterman 2006).

\subsection{Deficits in establishing structural syntactic dependencies}

Whereas Grodzinsky explicitly regards deficits in movement operations as causing the impaired comprehension of sentences with moved object NPs, other researchers have suggested that these comprehension problems are due to a deficit in establishing a structural relationship between the moved constituent and its trace (e.g. Mauner, Fromkin, and Cornell 1993). A deficit in establishing syntactic structural relationships should also affect other syntactic phenomena where a similar structural dependency holds. A case in point is the structural relation that holds between referentially dependent NPs and their antecedents. Van der Lely and colleagues were the first to investigate the comprehension of passives and referentially dependent NPs in children with Specific Language Impairment (van der Lely 1996, van der Lely and Stollwerck 1997). They found that children with Specific Language Impairment were impaired in 
interpreting passives and referentially dependent NPs and suggested that children with Specific Language Impairment suffer from a deficit with non-local structure-dependent relations that, for instance, impairs the computation of the binding domain. Van der Lely's Computational Grammatical Complexity Hypothesis (van der Lely 2005) posits a rather broad syntactic deficit that is not precisely couched in theoretical terms. It has been criticized for being too broad to capture more fine-grained deficits in Specific Language Impairment affecting, for instance, agreement but not tense inflection (cf. Clahsen 2008).

Interestingly, the deficits obtained for binding relationships might turn out to be specific for certain language disorders. Agrammatic Broca's aphasics and children with Specific Language Impairment display particular problems in interpreting pronouns and often accept a reading in which the pronoun is anaphorically linked to an antecedent. Thus, they might accept a sentence such as Mowgli is tickling him as a description of a picture depicting Mowgli tickling himself (Van der Lely and Stollwerck 1997; Grodzinsky et al. 1993). In doing so, these language impaired individuals mirror unimpaired children who also take considerable longer to correctly interpret non-reflexive pronouns compared to reflexive pronouns (cf. Guasti 2002: chapter 8 for overview).

A different picture emerged in two recent investigations on the comprehension of passives and anaphoric elements in individuals with Down syndrome (Ring and Clahsen 2005; Perovic 2006). These studies found a marked deficit in interpreting reflexive pronouns and passive sentences individuals with Down syndrome. In contrast, the interpretation of pronouns and active sentences was unimpaired (Ring and Clahsen 2005; Perovic 2006). In an attempt to provide a unitary account for the deficits in interpreting passives and reflexive pronouns, Ring and Clahsen (2005) have suggested that individuals with Down syndrome suffer from a specific syntactic deficit leading to the inability to construct $A$ (rgument)-chains. An A-chain requires that the two elements connected by the chain (the moved object NP and its trace, respectively the reflexive and its antecedent) are in the same local structural configuration, share the same syntactic features via coindexation, and the reflexive pronoun respectively the trace are ccommanded by the antecedent respectively the moved object NP (Chomsky 1995; Reuland 2001). A deficit in forming A-chains causes that a reflexive pronoun cannot be syntactically bound by its local antecedent. Likewise a moved object NP cannot be related to its trace resulting in defective theta-role assignment to the moved NP. Both will lead to comprehension problems. Ring and Clahsen also investigated a group of children with Williams syndrome who were matched for age and IQ with the Down syndrome subjects. Despite a similar impairment in intellectual development, the subjects with Williams syndrome did not show a deficit in the comprehension of passives or referentially dependent NPs such as reflexive pronouns. Ring and Clahsen thus suggest that the comprehension problems observed in individuals with Down syndrome are specific for this syndrome and cannot be explained by their impaired intellectual development. The claim that individuals with Down syndrome suffer from a specific syntactic deficit in constructing A-chains is also interpreted as being informative for theoretical accounts of binding. Thus, Perovic (2006) argues that the data support a fractionation of binding into a syntactic component governing the interpretation of bound anaphors and impaired in Down syndrome and an extra-syntactic component regulating the coreferential interpretation of pronouns and intact in individuals with Down syndrome.

The data and interpretations presented by Ring and Clahsen (2005) and Perovic (2006) are certainly fascinating. Note, however, that much hinges on whether or not the adapted syntactic interpretation of binding relations proves valid. Also, Ring and Clahsen admit that their interpretation does not capture every error pattern observed in their data. Thus, younger children with Down syndrome also committed significantly more reversal errors than unimpaired 
control children in interpreting active sentences. As active sentences are said to not involve Achains, this error pattern is unexplained by Ring and Clahsen's account. Finally, Ring and Clahsen (2005) and Perovic (2006) link the deficit in interpreting reflexive pronouns to the deficit in interpreting passive sentences and provide a syntactic explanation that relates these comprehension problems to an inability to construct A-chains. Note however, that while deficits in interpreting referentially dependent NPs seem to vary between different deficit syndromes, such variation can not be observed with respect to the comprehension of passives. Independent of the particular language disorder, language impaired subjects display similar problems in understanding passive clauses (cf. section 1.2.2.). This observation asks for an explanation. Are the similar problems in passive comprehension caused by different language deficits in different deficit syndromes? And if so what are these deficits that lead to similar performance in passive comprehension but to different performance in interpreting referentially dependent NPs? Further research is needed to evaluate these issues.

\section{Problems in accounting for syntactic deficits}

As indicated by these short sketches on syntactic deficit theories advocated in the field, to date the controversies about how to capture and explain language impairments that seem to affect syntactic representations have been going on. In these controversies, syntactic deficit theories have been challenged both by conflicting empirical data and by developments in syntactic theory that force an adaptation of deficit approaches to encompass the new evidence and the new developments in syntactic theory - a venture that often turns out to be quite intricate. Besides the challenges posed by new data and new theoretical developments, syntactic deficit accounts, such as those presented above, are also subject to more general discussions on the adequacy of deficit accounts that assume language impairments to be due to deficits in syntactic representations specific for a given syndrome.

The accounts presented in section 2 above propose a deficit in syntactic representations and thus a deficit in grammatical competence that underlies impaired language behaviour. Such a deficit in grammatical competence should affect all aspects of language performance in parallel (Weigl and Bierwisch 1970). A defective syntactic structure or function should no longer be produced, comprehended, or judged correctly by an affected individual. ${ }^{12}$

A crucial issue for such accounts is their all-or-none flavour. A specific syntactic structure or function is either intact or impaired and thus the language impaired speaker is or is not able to produce grammatical structures that involve the respective syntactic representation. Note, however, that such all-or-none behaviour is rarely observed in individuals. Grammatical competence is only one factor determining performance, with other factors, such as e.g. processing limitations, social, pragmatic, or discourse factors affecting it, too. Indeed, it has been shown that syntactic deficits, observed for instance in agrammatic Broca's aphasics, deteriorate or ameliorate depending on task demands or time constraints that lead to a lowering or raising of the processing load associated with the task (Kolk and Heeschen 1992). Tasks which minimize processing load, such as cloze tasks where the subject has to produce only the structure or word of interest whereas the sentential context is given, often lead to better performance than more unrestrained tasks (e.g. Kolk and Heeschen 1992). Other syntaxexternal factors that influence the performance of language impaired subjects relate to the testing situation, i.e. to factors such as the familiarity with the investigator or the formality of the testing situation (e.g. Heeschen and Schegloff 2003). All these factors contribute to the observation that language impaired individuals will only rarely achieve a correctness scores of $100 \%$ for a tested construction in an experimental investigation. 
What however is to be concluded from correctness scores of $80 \%, 60 \%, 40 \%$, or $20 \%$ ? That is, when is the correctness score low enough to conclude that a syntactic representation is defective and when is it high enough to conclude that syntactic representations are basically unimpaired? Consider as an example, data that come from an investigation on the production of wh-questions in German agrammatic Broca's aphasics (Neuhaus and Penke 2008). According to the Tree-Pruning Hypothesis (Friedmann and Grodzinsky 1997) individuals suffering from agrammatic Broca's aphasia should no longer succeed in producing grammatical wh-questions since the CP layer is pruned from syntactic representations (see section 2.2.2.). In an experiment eliciting wh-questions, the seven tested aphasic subjects succeeded in $66.1 \%$ of the contexts in producing a correct wh-question that unambiguously involved the projection of the CP layer, a score that was significantly lower than the $97.5 \%$ obtained by unimpaired control subjects (Neuhaus and Penke 2008). The significant difference between aphasic subjects and unimpaired control subjects indicates that the aphasic subjects suffer from an impairment in producing wh-questions. But is this impairment due to an inability to project $\mathrm{CP}$ as claimed by the Tree-Pruning Hypothesis? If this were the case, how then can we account for the observation that the aphasic subjects succeeded in $66.1 \%$ of the contexts in producing whquestions that required the projection of the CP layer?

A related problem comes from the variability in language behaviour a group of individuals suffering from the same language disorder will typically display. In the investigation of whquestion production abilities in German Broca's aphasics mentioned above (Neuhaus and Penke 2008), individual correctness scores of the seven tested aphasic subjects ranged from $27.8 \%$ to $96.3 \%$. Note in addition that the scores for the unimpaired control subjects in this task ranged between $88.3 \%$ and $100 \%$. Thus, rather than a clear boundary between impaired and spared performance, there seems to be a continuum between the performance of language unimpaired and impaired individuals. This continuum, often observed for impaired and unimpaired performance, and the variability in performance that typically holds in a group of language impaired individuals renders any strong claims on a representational deficit (such as the claim: 'agrammatic syntactic representations are pruned') problematic. As sketched in section 2.2.2., one solution to this problem of variability is to integrate the idea that language disorders are gradable and come in different levels of severity. This move, however, requires independent criteria to establish the severity of language disorder lest to avoid circularity in argumentation.

The data on wh-question production in German agrammatic aphasia presented above illustrate that in fact data of language impaired subjects are complex and difficult to interpret in practice. Their interpretation - as well as the conclusions drawn on the basis of such data depend crucially on issues in statistical methodology, subject sampling, and data analysis that have been at the heart of much discussion in the field (cf. e.g. Drai and Grodzinsky 2006; Grodzinsky 2000 and the discussions following these two articles for overview).

The problems in identifying all-or-none-behaviour in language impaired individuals have led to a second group of approaches accounting for language deficits. In contrast to representational syntactic-deficit accounts, processing accounts state that syntactic representations are not impaired in language disorders and can still be put to use. According to these accounts, the observed problems result from a deficit in language processing caused by limitations of the processing capacity. Such processing limitations may be domain-specific, i.e. restricted to the processing of language, or domain-general, affecting non-verbal processing alike. Processing accounts have suggested, for instance, that the language behaviour observed in language impaired subjects is caused by reductions in memory components such as working memory (e.g. Just and Carpenter 1992) or verbal short-term memory (e.g. Gathercole and 
Baddeley 1990). A consequence of such a deficit might be that language input can not be kept long enough in working memory to extract morpho-syntactic information, especially in complex sentences where for instance deviations of canonical ordering occur. Other researchers have suggested that processing limitations result from a restricted amount of energy available to the subject for a specific task (e.g. Lapointe 1985; Bates and Wulfeck 1989; Avrutin 2000) or from desynchronisation in the temporal course of language processing (e.g. Kolk 1995). In the former case, it might be too costly for a language impaired individual to compute specific syntactic constructions or relations. In the later case he/she might not be able to compute syntactic structure or structural relationships in a given temporal window, either because the computation is too slow or its results decay too quickly.

Limitations of processing capacity have been held responsible for the language impairments found in a wide variety of language deficits such as agrammatic Broca's aphasia (e.g. Bates and Wulfeck 1989; Kolk 1995; Caplan and Waters 1995), Wernicke's aphasia (e.g. Lavorel 1982), Specific Language Impairment (cf. Leonard 1998, Leonard et al. 2007 for overview), Down syndrome (e.g. Chapman, Hesketh, and Kistler 2002), and early unilateral focal brain lesions in children (Bates and Roe 2001).

A critical issue with respect to processing accounts is whether the proposed limitations in processing capacity cause the observed language impairments or whether they simply are a cosymptom that accompanies syntactic deficits in specific language disorders without causing them. Despite this issue, processing accounts are well suited to capture the continuum in performance that can be observed between impaired and unimpaired subjects and within groups of individuals suffering from the same language impairment. The concept that language performance, and thus also language deficits, are gradable comes naturally to processing accounts. Processing limitations can vary depending on the severity of the underlying disorder that affects the processing of syntactic structures or structural relationships. With growing processing costs or decreasing processing capacities the ability to implement the intact syntactic knowledge in language performance ameliorates or deteriorates. Processing-deficit accounts can, hence, also account for language behaviour that is neither completely impaired nor completely intact. And moreover, they provide an explanation for the observation, that the grammatical deficits observed in language impaired speakers deteriorate or ameliorate depending on task demands or time constraints that lead to a lowering or raising of the processing load (Kolk and Heeschen 1992) - a finding that is difficult to account for in representational-deficit theories which claim that syntactic representations are either intact or impaired.

The long-standing debate between representational-deficit accounts and processing-deficit accounts is one of the central debates in the field of language disorders and far from being settled. However, an integration of these two types of accounts might well turn out to be a fruitful third path in research on language disorders. Syntactic deficit accounts inform us where to expect problems in language impaired speakers. Thus, syntactic structures that involve the projection of fully-fledged syntactic trees or the interpretation of moved noun phrases which reverse canonical ordering of arguments are likely to cause problems for language impaired individuals. Performance deficit accounts help us in predicting when such a deficit is likely to appear, namely when processing load increases, for example because more syntactic operations have to be computed or the task is more taxing and exceeds the processing capacities of the individual subject. For an illustration of this point, consider again the data on wh-question production in German Broca's aphasics (Neuhaus and Penke 2008). Production of wh-questions was tested by an elicitation task, where subjects had to transform a given main clause into a wh-question, and a sentence repetition task, where subjects were asked to repeat 
wh-questions as accurately as possible. The repetition of a given syntactic structure is less demanding than the construction of this structure by the individual her/himself. A comparison of the correctness scores obtained in these two experiments yielded that the production of whquestions resulted in better performance in the less demanding repetition task (mean correctness score $78.6 \%$ ) than in the more demanding elicitation task (mean correctness score $66.1 \%$ ), a finding that illustrates the influence of task demands on language behaviour in language impaired individuals (cf. also Kolk and Heeschen 1992).

An interesting finding suggestive of the proposed integration between representationaldeficit accounts (where-accounts) and processing-deficit accounts (when-accounts) is the observation that syntax-related deficits are largely unspecific to a particular deficit syndrome. Rather than finding deficits that are characteristic for only one particular deficit syndrome, deficit symptoms such as omissions of free functional elements, problems with bound inflectional morphology, reduction of syntactic complexity, and problems in interpreting sentences with noncanonical argument order are found in a wide variety of acquired and developmental deficit syndromes (cf. section 1.2.). These symptoms seem to mark critical areas in the language system which are typically affected when an individual suffers from a limitation of her/his language ability. Syntactic (deficit) theories serve to inform us about the factors that make these critical areas vulnerable to language impairments. That independent of a particular deficit syndrome the same critical areas are affected and similar deficit symptoms occur throughout syndromes indicates a processing deficit. Whatever the underlying neural deficit, the deficit leads to limitations in processing capacities that affect the critical areas in the language system, resulting in similar deficit symptoms across syndromes.

\section{Summary and outlook}

The investigation of syntactic disorders is a fascinating topic where broad theoretical issues on the nature of the human language capacity and specific theoretical issues related to syntactic representations interact with empirical research conducted with a challenging subject group. A focus in the research on syntactic disorders is to find out if and how syntactic representations are defective in language impaired speakers. As indicated by the overview in section 2, syntactic theory and syntactic deficit approaches have co-evolved during the last 40 years. New developments in syntactic theory, such as the distinction between S(urface)-structure and $\mathrm{D}$ (eep)-structure, the Split-INFL Hypothesis, or the role ascribed to features, have prompted new developments in accounting for language disorders. To date researchers active in the field have not agreed on how to capture and explain syntax-related deficits for a single deficit syndrome. However, the controversies that have arisen in the field have undeniably advanced our knowledge on the language impairments observed in particular deficit syndromes. With new developments in syntactic theory and subsequent new suggestions how to capture syntaxrelated language impairments new facets of syntactic knowledge have come under scrutiny in language impaired individuals. Consequently, a growing body of data has been collected over the years on which aspects of syntax are impaired or retained in particular deficit syndromes. As is the case in science, each new piece of evidence has given rise to new and more specific questions to investigate and answer, in this way accumulating our knowledge on language deficits.

While syntactic theories and the advancements within theoretical approaches have been used to capture and explain language impairments, data of language impaired speakers have on the other hand only rarely been taken as evidence in advancing syntactic theories. However, this aspect of the investigation of language deficits certainly merits some research efforts. Insights in our language faculty can only be gained by the analysis of performance data 
reflecting the application of this knowledge. In our aim to elucidate the nature of the human language faculty, we should consider all sorts of evidence - including data that come from the investigation of language disorders. Data presented by theoretical linguists in advancing syntactic theories is no privileged type of data in this respect, but it constitutes just another type of performance data that can be used to investigate our language faculty (cf. Penke \& Rosenbach 2007). That errors occurring in the production or comprehension of particular linguistic structures or forms in language impaired individuals constitute a valuable type of data has, for instance, been shown in research on inflectional morphology. Here the selective vulnerability of regular respectively irregular inflectional morphology in specific language syndromes has been taken as evidence for a qualitative distinct representation of regular and irregular inflection in the human mind/brain (cf. Pinker 1999; Penke 2006 for overview). Also, the finding that regular and irregular inflection often dissociate in language disorders can be used as diagnostic for which inflectional markers are regular or irregular in a particular language (cf. Penke \& Krause 2002, Penke 2008). Research that does not only take syntactic theory as the basis for explaining language deficits, but which aims in advancing syntactic theory based on error data or breakdown patterns observed in language impaired individuals might prove to be a valuable data type in investigating the nature of syntactic representations.

\section{References}

Avrutin, Sergey 2000 Comprehension of discourse-linked and non-discourse-linked questions by children and Broca's aphasics. In Yosef Grodzinsky, L. Shapiro, and D. Swinney (eds.), Language and the Brain: Representation and Processing, 295-313. San Diego: Academic Press.

Baker, M. 1985 The mirror principle and morphosyntactic explanation. Linguistic Inquiry 16, 373-416.

Bartke Susanne and Julia Siegmueller (eds.)2004 Williams Syndrome across Languages. Amsterdam: Benjamins.

Bartolucci, G., S.J. Pierce, and D. Streiner 1980 Cross-sectional studies of grammatical morphemes in autistic and mentally retarded children. Journal of Autism and Developmental Disorders 10(1): 39-50.

Bastiaanse, Roelien and Susan Edwards 2004 Word order and finiteness in Dutch and English Broca's and Wernicke's aphasia. Brain and Language 89: 91-107.

Bates, Elisabeth, Angela Friederici, and Beverly Wulfeck 1987 Grammatical morphology in aphasia. Cortex 23: 545-574.

Bates, Elisabeth, Angela Friederici, Beverly Wulfeck, and L.A. Juarez1988 On the preservation of word order in aphasia: cross-linguistic evidence. Brain and Language 33: 323363. 
Bates, Elisabeth and Beverly Wulfeck 1989 Crosslinguistic study of aphasia. In: Brian MacWhinney and Elisabeth Bates (eds.), The Crosslinguistic Study of Sentence Processing, 328-374. Cambridge: Cambridge University Press.

Bates, Elisabeth and K. Roe 2001 Language development in children with unilateral brain injury. In: C.A. Nelson and M. Luciana (eds.), Handbook of Developmental Cognitive Neuroscience, 281-307. Cambridge, MA: MIT Press.

Bellugi, Ursula and Marie St. George (eds.) 2000 Linking Cognitive Neuroscience and Molecular Genetics: New Perspectives from Williams Syndrome. Special issue of Journal of Cognitive Neuroscience 12: Supplement.

Bellugi, Ursula, L. Lichtenberger, W. Jones, and Z. Lai 2000 The neurocognitive profile of Williams syndrome. A complex pattern of strenghts and weaknesses. Journal of Cognitive Neuroscience 12 (Supplement): 7-29.

Berndt, R. S., C.C. Mitchum, and A.N. Haendiges 1996 Comprehension of reversible sentences in "agrammatism": a meta-analysis. Cognition 58: 289-308.

Caplan, David 1985 Syntactic and semantic structures in agrammatism. In: Marie Louise Kean (ed.), Agrammatism, 125-152. Orlando: Academic Press.

Caplan, David and G. Waters 1995 Aphasic disorders of syntactic comprehension and working memory capacity. Cognitive Neuropsychology 12: 637-651.

Caramazza, Alfonso1984 The logic of neuropsychological research and the problem of patient classification in aphasia. Brain and Language 21: 9-20.

Caramazza, Alfonso1992 Is cognitive neuropsychology possible? Journal of Cognitive Neuroscience 4(1): 80-95.

Caramazza, Alfonso and Edgar Zurif1976 Dissociation of algorhithmic and heuristic processes in language comprehension: evidence from aphasia. Brain and Language 3: 572582.

Caramazza, Alfonso and Rita Berndt 1978 Semantic and syntactic processes in aphasia: a review of the literature. Psychological Bulletin 85: 898-918.

Chapman, R.S., L.J. Hesketh, and D.E. Kistler 2002 Predicting longitudinal change in language production and comprehension in individuals with Down syndrome: hierarchical linear modelling. Journal of Speech, Language, and Hearing Research 45: 902-915.

Chomsky, Noam 1957 Syntactic Structures. The Hague: Mouton.

Chomsky, Noam 1980 Rules and representations. Behavioral and Brain Sciences 3: 1-14.

Chomsky, Noam 1981 Lectures on Government and Binding. Dordrecht: Foris. 
Chomsky, Noam 1995 The Minimalist Program. Cambridge, MA: MIT Press.

Chomsky, Noam 2000 Minimalist inquiries: the framework. In: R. Martin, D. Michaelis, \& Juan Uriagereka (eds.), Step by step, 89-155. Cambridge MA: MIT Press.

Chomsky, Noam 2002 On Nature and Language. Cambridge: Cambridge University Press.

Clahsen, Harald2008 Chomskyan syntactic theory and language disorders. In: Martin Ball, Michael Perkins, Nicole Mueller, and Sara Howard (eds.), The Handbook of Clinical Linguistics, 165-183. Oxford: Blackwell.

Clahsen, Harald, Susanne Bartke, and Sandra Göllner 1997 Formal features in impaired grammars: a comparison of English and German SLI. Journal of Neurolinguistics 10 (2/3): 151171.

Clahsen, Harald and Mayella Almazan 1998 Syntax and morphology in Williams syndrome. Cognition 68: 167-198.

Curtiss, Susan and Jeannette Schaeffer 2005 Syntactic development in children with hemispherectomy: the I-, D-, and C-systems. Brain and Language 94: 147-166.

De Bleser, Ria and Josef Bayer 1991 On the role of inflectional morphology in agrammatism. In: Michael Hammond (ed.), Theoretical Morphology: Approaches in Modern Linguistics, 45-69. San Diego: Academic Press.

Dennis, M. and H. Whitaker 1976 Language acquisition following hemidecortication. Brain and Language 3: 404-433.

Dick, Frederic, Beverly Wulfeck, Magda Krupa-Kwiatkowski, and Elizabeth Bates 2004 The development of complex sentence interpretation in typically developing children compared with children with specific language impairments or early unilateral focal lesions. Developmental Science 7(3): 360-377.

Drai Dan and Yosef Grodzinsky 2006 A new empirical angle on the variability debate: Quantitative neurosyntactic analyses of a large data set from Broca's Aphasia. Brain and Language 96: 117-128.

Dromi, E., Laurence Leonard, G. Adam, and S. Zadunaisky-Ehrlich 1999 Verb agreement morphology in Hebrew speaking children with specific language impairment. Journal of Speech and Hearing Research 42: 1414-1431.

Eadie, P.A., M.E. Fey, J.M. Douglas, and C.L. Parsons 2002 Profiles of grammatical morphology and sentence imitation in children with Specific Language Impairment and Down syndrome. Journal of Speech, Language, and Hearing Research 45: 720-732.

Edwards, Susan 2005 Fluent Aphasia. Cambridge: Cambridge University Press. 
Edwards, Susan and David Lightfoot2000 Intact grammars but intermittent access. Behavioral and Brain Sciences 23(1): 31-32.

Fanselow, Gisbert and Sascha Felix 1987 Sprachtheorie: Eine Einführung in die generative Grammatik. [Linguistic Theory: An Introduction into Generative Grammar, Volume II]. Tübingen: Francke.

Faroqi-Shah, Yasmeen and Cynthia K. Thompson 2004 Semantic, lexical, and phonological influences on the production of verb inflections in agrammatic aphasia. Brain and Language 89: 484-498.

Fodor, Jerry A. 1983 The Modularity of Mind. Cambridge, MA: MIT Press.

Friedmann, Naama and Yosef Grodzinsky 1997 Tense and agreement in agrammatic production: pruning the syntactic tree. Brain and Language 56: 397-425.

Friedmann, Naama and R. Novogrodsky 2004 The acquisition of relative clause comprehension in Hebrew: a study of SLI and normal development. Journal of Child Language 31, 661-681.

Friedmann, Naama and Ronit Szterman 2006 Syntactic movement in orally trained children with hearing impairment. Journal of Deaf Studies and Deaf Education 11(1): 56-75.

Fromkin, Victoria A. 1971 The non-anomalous nature of anomalous utterances. Language 47(1): 27-52.

Fromkin, Victoria A. 1997 Some thoughts about the brain/mind/language interface. Lingua 100: 3-27.

Gathercole, S. and A. Baddeley 1990 Phonological memory deficits in language impaired children: is there a causal connection? Journal of Memory and Language 29: 336-360.

Goodglass, Harold, Julie Ann Christiansen, and Roberta Gallagher 1993 Comparison of morphology and syntax in free narrative and structured tests: fluent vs. nonfluent aphasics. Cortex 29: 377-407.

Gopnik, Myrna and M.B. Crago 1991 Familial aggregation of a developmental language disorder. Cognition 39: 1-50.

Grober, E. and S. Bang 1995 Sentence comprehension in Alzheimer's disease. Developmental Neuropsychology 11: 95-107.

Grodzinsky, Yosef 1984 The syntactic characterization of agrammatism. Cognition 16: 99120.

Grodzinsky, Yosef 1990 Theoretical Perspectives on Language Deficits. London: MIT Press. 
Grodzinsky, Yosef 1995 Language 50: 27-51.

A restrictive theory of agrammatic comprehension. Brain and

Grodzinsky, Yosef 2000 The neurology of syntax. Behavioral and Brain Sciences 23(1): 171.

Grodzinsky, Yosef, Amy Pierce, and Susan Marakovitz 1991 Neuropsychological reasons for a transformational derivation of syntactic passive. Natural Language and Linguistic Theory 9(3): 431-453.

Grodzinsky, Yosef, Ken Wexler, Yu-Chin Chien, Susan Marakovitz and Julie Solomon The breakdown of binding relations. Brain and Language 45: 396-422.

1993

Grodzinsky, Yosef and L. Finkel 1998 The neurology of empty categories: aphasics' failure to detect ungrammaticality. Journal of Cognitive Neuroscience 10(2): 281-292.

Guasti, Maria Teresa 2002 Language Acquisition. Cambridge MA: MIT Press.

Haegeman, Liliane 1991 Introduction to Government and Binding Theory. Oxford: Blackwell.

Hagiwara, H. 1995 The breakdown of functional categories and the economy of derivation. Brain and Language 50: 92-116.

Hamann, Cornelia 2006 Speculations about Early Syntax: The production of Wh-questions by normally developing French children and French children with SLI". Catalan Journal of Linguistics 5: 143-189.

Hamann, Cornelia, Laurice Tuller, Cécile Monjauze, Hélène Delage, and Célia Henry 2007

(Un)successful subordination in French-speaking children and adolescents with SLI. In: Heather Caunt-Nutton, Samantha Kulatilake, and I-Hao Woo (eds.), Proceedings of BUCLD 31. Somerville: Cascadilla Press.

Heeschen, Claus and Emanuel Schegloff 2003 Aphasic agrammatism as interactional artefact and achievement. In: Charles Goodwin (ed.), Conversation and Brain Damage, 231282. Oxford: Oxford University Press.

Heilman, K. and R.J. Scholes 1976 The nature of comprehension errors in Broca's, conduction and Wernicke's aphasics. Cortex 12: 258-265.

Hickok, Gregory and Sergey Avrutin 1995 Representation, referentiality, and processing in agrammatic comprehension: two case studies. Brain and Language 50: 10-26.

Janssen, Ulrike and Martina Penke 2002 How are inflectional affixes organized in the mental lexicon? Evidence from the investigation of agreement errors in agrammatic aphasics. Brain and Language 81: 180-191. 
Johnston, J.R. 1994 Cognitive abilities of language-impaired children. In: Ruth Watkins and Mabel Rice (eds.), Specific Language Impairments in Children: Current Directions in Research and Intervention, 107-121. Baltimore: Brookes.

Just, M. and P. Carpenter 1992 A capacity theory of comprehension: individual differences in working memory. Psychological Review 98: 122-149.

Karmiloff-Smith, A. 1998 Development itself is the key to understanding developmental disorders. Trends in Cognitive Sciences 2: 389-398.

Kernan, Keith and Sharon Sabsay 1996 Linguistic and cognitive ability of adults with Down syndrome and mental retardation of unknown etiology. Jounal of Communication Disorders 29: 401-421.

Kolk, Herman 1995 A time-based approach to agrammatic production. Brain and Language 50: 282-303.

Kolk, Herman and Claus Heeschen 1992 Agrammatism, paragrammatism and the management of language. Language and Cognitive Processes 7: 89-129.

Koopman, H. and D. Sportiche 1988 The position of subjects. Lingua 85: 211-58.

Lai, C. S., S.E. Fisher, J.A. Hurst, F. Vargha-Khadem, and A.P. Monaco 2001 A fork-headdomain gene is mutated in a severe speech and language disorder. Nature 413: 519-523.

Lapointe, S.G. 1985 A theory of verb form use in the speech of agrammatic aphasics. Brain and Language 24: 100-155.

Lavorel, P.M. 1982 Production strategies: a systems approach to Wernicke's aphasia. In: Michael Arbib, David Caplan and John C. Marshall (eds.), Neural Models of Language Processing, 135-164. New York: Academic Press.

Laws, G. and Dorothy Bishop 2003 A comparison of language abilities in adolescents with Down syndrome and children with Specific Language Impairment. Journal of Speech, Language, and Hearing Research 46: 1324-1339.

Leonard, Laurence 1995 Functional categories in the grammars of children with specific language impairment. Journal of Speech and Hearing Research 38: 1270-1283.

Leonard, Laurence 1998 Children with Specific Language Impairment. Cambridge, MA: MIT Press.

Leonard, Laurence, Susan Weismer, Carol Miller, David Francis, Bruce Tomblin, and Robert Kail2007 Speed of processing, working memory, and language impairment in children. Journal of Speech, Language, and Hearing Research 50: 408-428.

Levi Yonata and G. Kavé 1999 Language breakdown and linguistic theory: a tutorial overview. Lingua 107: 95-143. 
Levy Yonata and Jeannette Schaeffer (eds.) 2002 Language Competence across Populations - Towards a Definition of Specific Language Impairment in Children. Mahwah, NJ: Lawrence Erlbaum.

Liégeois, F., T. Baldeweg, A. Connelly, D.G. Gadian, M. Mishkin, and F. Vargha-Khadem 2003 Language fMRI abnormalities associated with FOXP2 gene mutation. Nature Neuroscience 6(11): 1230-1237.

Lonzi, L. and Claudio Luzzatti 1993 Relevance of adverb distribution for the analysis of sentence representation in agrammatic patients. Brain and Language 45: 306-317.

Marin, O.S.M., E.M. Saffran, and M.F. Schwartz 1976 Dissociations of language in aphasia: implications for normal function. In: S.R. Harnad, H.D. Steklis, and J. Lancaster (eds.), Origins and Evolution of Language and Speech, 868-884. New York: Academy of Sciences.

Mauner, G. Victoria Fromkin, and T.L. Cornell 1993 Comprehension and acceptability judgements in agrammatism: disruptions in the syntax of referential dependency. Brain and Language 45: 340-370.

Meaburn E., P.A. Dale, W. Craig, and R. Plomin 2002 Language-impaired children: no sign of the FOXP2 mutation. Neuroreport 13(8): 1075-1077.

Menn, Lise and Loraine K. Obler1990a Cross-language data and theories of agrammatism. In: Lise Menn and Loraine K. Obler (eds.), Agrammatic Aphasia: A Cross-Language Narrative Sourcebook, Vol. III, 1369-1389. Amsterdam: Benjamins.

Menn, Lise and Loraine K. Obler (eds.) 1990b Agrammatic Aphasia: A Cross-Language Narrative Sourcebook, Vol. I-III. Amsterdam: Benjamins.

Neuhaus, Eva and Martina Penke 2008 Production and comprehension of wh-questions in German Broca's aphasia. Journal of Neurolinguistics 21: 150-176.

Niemi, Jussi and Matti Laine 1997 Syntax and inflectional morphology in aphasia: quantitative aspects of Wernicke speakers narratives. Journal of Quantitative Linguistics 4(1-3): 181-189.

Ouhalla, Jamal 1993 Functional categories, agrammatism and language acquisition. Linguistische Berichte 143: 3-36.

Penke, Martina 1998 Die Grammatik des Agrammatismus: Eine linguistische Analyse zu Wortstellung und Flexion bei Broca-Aphasie [The Grammar of Agrammatism: A Linguistic Analysis of Word Order and Inflection in Broca's Aphasia]. Tübingen: Niemeyer.

Penke, Martina 2000 Unpruned trees in German Broca's aphasia. Behavioral and Brain Sciences 23(1): 46-47.

Penke, Martina 2001 Controversies about CP: a comparison of language acquisition and language impairments in Broca's aphasia. Brain and Language 77: 351-363. 
Penke, Martina 2006 Flexion im mentalen Lexikon [Inflectional Morphology in the Mental Lexicon]. Tübingen: Niemeyer.

Penke, Martina 2008 Morphology and language disorder. In: Martin Ball, Michael Perkins, Nicole Mueller and Sara Howard (eds.), The Handbook of Clinical Linguistics, 212-227. Oxford: Blackwell.

Penke, Martina and Marion Krause 2002 German noun plurals - a challenge to the DualMechanism Model. Brain and Language 81: 303-311.

Penke, Martina and Anette Rosenbach 2007 What counts as evidence in linguistics? In: Martina Penke and Anette Rosenbach (eds.), What Counts as Evidence in Linguistics - the Case of Innateness, 1-50. Amsterdam: John Benjamins.

Perovic, Alexandra 2006 Syntactic deficit in Down syndrome: more evidence for the modular organisation of language. Lingua 116: 1616-1630.

Pinker, Steven 1999 Words and Rules. New York: Basic Books.

Pollock, Jean-Yves 1989 Verb movement, Universal Grammar, and the structure of IP. Linguistic Inquiry 20: 365-424.

Reuland, E. 2001 Primitives of binding. Linguistic Inquiry 32: 439-492.

Rice, Mabel L. and Ken Wexler 1996 Towards tense as a clinical marker of specific language impairment in English-speaking children. Journal of Speech and Hearing Research 39: $1239-1257$.

Ring, Melanie and Harald Clahsen 2005 Distinct patterns of language impairment in Down's syndrome and Williams syndrome: the case of syntactic chains. Journal of Neurolinguistics 18: 479-501.

Rondal, J. and A. Comblain 1996 Language in adults with Down syndrome. Down Syndrome Research and Practice 4(1): 3-14.

Rugg, M.D. 1999 Functional neuroimaging in cognitive neuroscience. In: Colin Brown and Peter Hagoort (eds.), The Neurocognition of Language, 15-36. Oxford: Oxford University Press.

Schaeffer, Jeannette 2000 Aphasia research and theoretical linguistics guiding each other. Behavioral and Brain Sciences 23(1): 50-51.

Shallice, T. 1988 From Neuropsychology to Mental Structure. Cambridge: Cambridge University Press.

Shattuck-Hufnagel, Stefanie 1979 Speech errors as evidence for a serial order mechanism in sentence production. In: W.E. Cooper and E.C.T. Walker (eds.), Sentence Processing: 
Psycholinguistic Studies Presented to Merrill Garrett, 295-342. Hillsdale, NJ: Lawrence Erlbaum.

SLI Consortium 2002 A genomewide scan identifies two novel loci involved in specific language impairment. American Journal of Human Genetics 70: 384-398.

Stromswold, Karen 2001 The heritability of language: a review and metaanalysis of twin, adoption, and linkage studies. Language 77(4): 647-723.

Tager-Flusberg, Helen 1981 Sentence comprehension in autistic children. Applied Psycholinguistics 2: 5-24.

Tager-Flusberg, Helen 2002 Language impairment in children with complex neurodevelopmental disorders: the case of Autism. In: Yonata Levy and Jeannette Schaeffer (eds.), Language Competence across Populations - Towards a Definition of Specific Language Impairment in Children, 297-321. Mahwah, NJ: Lawrence Erlbaum.

Tager-Flusberg Helen, Susan Calkins, Tina Nolin, Therese Baumberger, Marcia Anderson, and Ann Chudwick-Dias 1990 A longitudinal study of language acquisition in autistic and Down syndrome children. Journal of Autism and Developmental Disorders 20(1): 1-21.

Tallal P. $1990 \quad$ Fine-grained discrimination deficits in language-learning impaired children are specific neither to the auditory modality nor to speech perception. Journal of Speech and Hearing Research 33: 616-621.

Teuber, H.-L. 1955 Physiological psychology. Annual Review of Psychology 9: 267-296.

Thal, D., S. Tobias, and D. Morrison 1991 Language and gesture in late talkers: a one-year follow-up. Journal of Speech and Hearing Research 34(3): 604-612.

Townsend, J., Beverly Wulfeck, S. Nichols, and L. Koch 1995 Attentional deficits in children with developmental language disorder. Technical Report CND-9503. Center for Rerearch in Language, University of California at San Diego.

Tsimpli, lanthi Maria2001 LF-interpretability and language development: A study of verbal and nominal features in Greek normally developing and SLI children. Brain and Language, 77: 432-448.

van der Lely, Heather 1996 Specifically language impaired and normally developing children: verbal passive vs. adjectival passive sentence interpretation. Lingua 98: 243-272.

van der Lely, Heather 2005 Domain-specific cognitive systems: insight from GrammaticalSLI. TRENDS in Cognitive Sciences 9(2): 53-59.

van der Lely, Heather and L. Stollwerck 1997 Binding theory and specifically language impaired children. Cognition 62: 245-290. 
van der Lely, Heather and Michael T. Ullman2001 Past tense morphology in specifically language impaired and normally developing children. Language and Cognitive Processes 16(2/3): 177-217.

Wang, Paul and Ursula Bellugi 1993 Williams syndrome, Down syndrome, and cognitive neuroscience. American Journal of Diseases of Children 147: 1246-1251.

Weigl, E. and Manfred Bierwisch 1970 Neuropsychology and linguistics: topics of common research. Foundations of Language 6: 1-18.

Wenzlaff, Michaela and Harald Clahsen 2004 Tense and agreement in German agrammatism. Brain and Language 89: 57-68.

Wexler, Ken 1994 Optional infinitives, head movement and economy of derivation. In: Norbert Hornstein and David Lightfoot (eds.), Verb Movement, 305-350. Cambridge: Cambridge University Press.

Wexler, Ken, C. Schütze, and Mabel Rice 1998 Subject case in children with SLI and unaffected controls: evidence for the Agr/Tns omission model. Language Acquisition 7: 317344. 


\section{Appendix}

\section{Broca's aphasia}

Broca's aphasia is an acquired language disorder that is typically caused by strokes affecting anterior parts of the left hemisphere. Spontaneous speech production of Broca's aphasics displays a symptom called Agrammatism. Agrammatic speech is characterized by the following signs: (i) omission of free function words, (ii) problems with bound inflectional morphology leading to omissions and/or substitutions (see Penke 2008 for overview), (iii) reduction of sentence length, leading to the preponderence of one or two word utterances in spontaneous speech, (iv) occurrence of root-clause infinitives, and (v) reduced syntactic complexity, resulting in a preponderence of canonical sentence structures and a lack of more complex structures such as subordinate clauses, questions, or utterances with a topicalised constituent. In language comprehension, most Broca's aphasics display a better understanding of sentences with a canonical word order compared to sentences with non-canonical word order where the object has moved out of its base-generated position and precedes the subject.

Major controversies are concerned with the issue whether or not Agrammatism is due to a deficit in syntactic competence, and if yes, how to account for this deficit within a theoretical approach (see sections 2. and 3.).

Suggested reading: Menn and Obler (1990b); Penke (1998); Grodzinsky (2000)

\section{Specific Language Impairment}

Specific Language Impairment is a cover term for delays respectively disorders of the normal acquisition of language that cannot be attributed to obvious neurological, cognitive, psychoemotional or significant hearing impairments. Consequently, Specific Language Impairment has proven to be a heterogeneous disorder and has been divided into syndrome subgroups. Relevant for the issue of syntactic deficits is the syndrome subgroup called Grammatical Specific Language Impairment that is characterised by problems in the acquisition of morphosyntax. Core symptoms of Grammatical Specific Language Impairment include impairments with inflectional morphology and difficulties with producing or interpreting complex sentences, such as subordinate clauses, relative clauses, or passives.

Whether the language impairments in Grammatical Specific Language Impairment are due to a representational deficit affecting syntactic structures or relationships and if so how to account for them (cf. section 2) or whether the observed impairments are due to a processing deficit that affects language processing capacities (cf. Leonard 1998) is a major issue of controversy in the field. Another central debate concerns the issue whether or not the language deficits of children with SLI are language-specific, or whether they can be attributed to domainunspecific cognitive impairments, such as deficits in rapid auditory discrimination, in symbolic play, in nonverbal attention, or in spatial imagery (cf. Tallal 1990; Thal, Tobias, and Morrison 1991; Johnston 1994; Townsend et al. 1995). Note, however, that the crucial issue here is not whether children with SLI have any non-linguistic cognitive deficits, but whether these deficits can explain the observed language impairments.

Suggested reading: Leonard (1998); Levy and Kavé (1999); Levy and Schaeffer (2002); Van der Lely (2005) 


\section{Williams syndrome}

Williams syndrome is a rare neurodevelopmental disorder of genetic origin that is associated with physical (e.g. renal and cardiovascular) anomalies and cognitive deficits, especially in nonlinguistic domains such as visuospatial constructive cognition.

One of the central controversies in the investigation of Williams syndrome concerns whether or not there is a dissociation between grammatical knowledge and other cognitive skills in individuals with this syndrome. Children with Williams syndrome show normal language skills with respect to regular inflectional morphology and the production respectively comprehension of complex sentence structures (e.g. subordinate clauses, passive clauses, tag questions, anaphors) despite severe mental retardation (Bellugi et al. 2000; Clahsen and Almazan 1998). The purported dissociation between grammar skills and general cognitive capacities observed in this disorder has been taken as evidence that the human language faculty is autonomous from other cognitive domains and that the properties of the language faculty cannot be put down to the operation of domain-general principles (see Bellugi et al. 2000). This view has, however, been challenged by researchers who dispute the autonomy of language from general cognitive capacities. These researchers have tried to show that grammatical abilities are not spared in affected children and, hence, are not dissociated from general cognitive capacities (KarmiloffSmith 1998). Moreover, it has been suggested that children with Williams syndrome undergo a different neural and cognitive development, resulting in the construction of a qualitatively different grammatical system in the brain. Note that if the grammatical system of individuals with Williams syndrome were indeed qualitatively different from the 'normal' system nothing could be concluded on the basis of data from Williams syndrome about the 'normal' language faculty, for instance about its autonomy from general cognition (Karmiloff-Smith 1998).

Suggested reading: Bellugi and St George (eds.) 2000; Bartke and Siegmueller (eds.) (2004)

\section{Down syndrome}

Down syndrome is a congenital neurodevelopmental disorder caused by a third copy of chromosome 21 that leads to moderate mental retardation and characteristic physiological traits. The language deficits of individuals with Down syndrome display similar characteristics to deficits observed in Specific Language Impairment. In speech production free grammatical morphemes are often omitted (Eadie et al. 2002) and the production of bound grammatical morphemes is impaired (Laws and Bishop 2003). In language comprehension, deficits arise in the comprehension of passive sentences and in establishing grammatical binding relations between anaphors and their referents (Ring and Clahsen 2005; Perovic 2006).

A critical issue regarding the language deficits in Down syndrome is whether the general limitation of cognitive capacities in individuals with Down syndrome is also responsible for the observed language deficits. If this were the case, claims regarding the modularity and autonomy of language capacities from general cognitive capacities (cf. Fodor 1983) would be severely threatened. To investigate this issue, language capacities of individuals with Down syndrome are compared to language capacities of individuals who have a similar level in nonverbal cognitive development, without suffering from Down syndrome. These can be unimpaired younger children or children with mental retardations due to a different neurodevelopmental disorder, such as individuals with Williams syndrome. A language performance that differs quantitatively and/or qualitatively from the performance of mental-age-matched controls is seen as indicative of an indigenous language deficit because the deficit surpasses a level that could be accounted for by the level of cognitive development. Especially the differences in cognitive capacities and deficits that are observed in individuals with Down syndrome and individuals with 
Williams syndrome indicate that language capacities are independent from general cognitive capacities since a comparable general cognitive impairment can lead to syndrome specific language deficits in these two syndromes.

Suggested reading: Wang and Bellugi (1993); Kernan and Sabsay (1996) 


\section{Notes}

1 Whenever the term 'Specific Language Impairment' is used in the text, this term refers to the subgroup of this deficit syndrome classified as Grammatical Specific Language Impairment (cf. Appendix).

2 Although the validity of these assumptions has been questioned by some, they nevertheless have proven very fruitful in linguistic investigations of language disorders. The major justification for adopting these axioms comes from the observation that results obtained from the analysis of language deficits have been supported by converging results coming from theoretical linguistics and from data obtained with other psycho- or neurolinguistic methodologies (see Caramazza 1992; Penke 2006: §2.3 for discussion).

3 Note that dissociations do not necessarily imply distinct mental representations of impaired respectively unimpaired entities or functions, since they can also be captured in systems that assume a unitary representation of these entities/functions (confer Shallice 1988; Penke 2006: $\S 2.3$ for discussion). However, if a distinct representation is postulated for two different linguistic entities/functions on theoretical grounds, than language disorders should be found that selectively affect one of the theoretically postulated entities/functions, but not the other.

$4 \quad$ The term double dissociation (Teuber 1955) describes the following situation: for two functions $X$ and $Y$, deficit syndrome $A$ impairs $X$ and spares $Y$, whereas deficit syndrome $B$ spares $X$ and impairs $Y$ (cf. Shallice 1988; Penke 2006: §2.3 for discussion).

5 Note, however, that individuals with Williams syndrome do not seem to display such problems (Clahsen and Almazan 1998).

6 The figure depicts the standard double-movement analysis of German V2 movement (cf. Fanselow and Felix 1987; Haegeman 1991). In main clauses, the verb starts out in the VP-final V position and successively moves over the INFL node, where it incorporates its finite inflectional morphology, to the $C$ position. The subject moves to SpeclP to realize its nominative case features and to enter into an agreement relation with the verb. In addition, the subject or any other constituent has to move to SpecCP to ensure that the finite verb ends up in the second structural sentence position (SVX or XVS word order).

7 The current version of the Extended Optional Infinitive Hypothesis has been reformulated to encompass underspecification of the tense and/or the agreement features (Wexler, Schütze, and Rice 1998). See Clahsen (2008) for discussion.

8 See Tsimpli (2000) for a broader deficit account which assumes a deficit in the accessibility of all uninterpretable features - not just the verb's phi-features - in individuals with Specific Language Impairment.

9 Grodzinsky and colleagues have suggested that the dissociation between impaired comprehension of passives and spared comprehension of actives observable in agrammatic aphasia argues for a syntactic approach to capture passives as developed in the framework of Government and Binding and discussed in the text. They argue that syntactic frameworks such as Lexical Functional Grammar or Generalised Phrase Structure Grammar where the derivation of passives does not involve movement but a lexical derivation can not account for this very circumscribed deficit in agrammatic comprehension. Hence, these latter approaches are considered not to be breakdown compatible and inferior to the framework of Government and Binding (cf. Grodzinsky, Pierce, and Marakovitz 1991).

10 A different theoretically based challenge is posed by the fact that the recent framework of the Minimalist Program discards with movement operations which Grodzinsky explicitly sees at the basis of agrammatic comprehension problems (cf. Edwards and Lightfoot 2000).

11 Because of the challenges the integration of the VP-Internal Subject Hypothesis poses to the TraceDeletion Hypothesis, Schaeffer (2000) has proposed to take the dissociation between impaired 
comprehension of sentences including object traces and spared comprehension of sentences with subject traces in agrammatic aphasia as evidence that the VP-Internal Subject Hypothesis is not valid.

12 Note that Grodzinsky (2000) has advocated the view that language production and language comprehension might be differently affected in Agrammatism. Whereas he assumes syntactic representations to be pruned in production, comprehension is claimed to suffer from the deletion of traces in syntactic representations. Such a move, however, presupposes the existence of two independent syntactic representations that are targeted by different language processes (comprehension vs. production) and that can be selectively affected in language disorders - a picture that deviates from the standard Chomskyan conception of an abstract grammatical competence that is put to use in language production and comprehension (Chomsky 1980). 